\title{
Review \\ The Exoscope in Neurosurgery: An Overview of the Current Literature of Intraoperative Use in Brain and Spine Surgery
}

\author{
Nicola Montemurro ${ }^{1, *(D)}$, Alba Scerrati ${ }^{2}\left(\mathbb{D}\right.$, Luca Ricciardi $^{3}(\mathbb{C})$ and Gianluca Trevisi ${ }^{4}(\mathbb{C}$ \\ 1 Department of Neurosurgery, Azienda Ospedaliera Universitaria Pisana (AOUP), University of Pisa, \\ 56100 Pisa, Italy \\ 2 Department of Neurosurgery, Sant'Anna University Hospital, 44124 Ferrara, Italy; scrlba@unife.it \\ 3 Department of NESMOS, Neurosurgery, Sant'Andrea Hospital, "Sapienza" University of Rome, \\ 00189 Rome, Italy; ricciardi.lu@gmail.com \\ 4 Department of Neurosurgery, Presidio Ospedaliero Santo Spirito, 65124 Pescara, Italy; \\ trevisi.gianluca@gmail.com \\ * Correspondence: nicola.montemurro@unipi.it
}

check for updates

Citation: Montemurro, N.; Scerrati, A.; Ricciardi, L.; Trevisi, G. The Exoscope in Neurosurgery: An Overview of the Current Literature of Intraoperative Use in Brain and Spine Surgery. J. Clin. Med. 2022, 11, 223. https://doi.org/10.3390/jcm11010223

Academic Editor: Morgan Broggi

Received: 27 November 2021

Accepted: 30 December 2021

Published: 31 December 2021

Publisher's Note: MDPI stays neutral with regard to jurisdictional claims in published maps and institutional affiliations.

Copyright: () 2021 by the authors. Licensee MDPI, Basel, Switzerland. This article is an open access article distributed under the terms and conditions of the Creative Commons Attribution (CC BY) license (https:// creativecommons.org/licenses/by/ $4.0 /)$.

\begin{abstract}
Background: Exoscopes are a safe and effective alternative or adjunct to the existing binocular surgical microscope for brain tumor, skull base surgery, aneurysm clipping and both cervical and lumbar complex spine surgery that probably will open a new era in the field of new tools and techniques in neurosurgery. Methods: A Pubmed and Ovid EMBASE search was performed to identify papers that include surgical experiences with the exoscope in neurosurgery. PRISMA guidelines (Preferred Reporting Items for Systematic Reviews and Meta-analyses) were followed. Results: A total of 86 articles and 1711 cases were included and analyzed in this review. Among 86 papers included in this review $74(86 \%)$ were published in the last 5 years. Out of 1711 surgical procedures, $1534(89.6 \%)$ were performed in the operative room, whereas $177(10.9 \%)$ were performed in the laboratory on cadavers. In more detail, 1251 (72.7\%) were reported as brain surgeries, whereas 274 $(16 \%)$ and $9(0.5 \%)$ were reported as spine and peripheral nerve surgeries, respectively. Considering only the clinical series (40 studies and 1328 patients), the overall surgical complication rate was $2.6 \%$ during the use of the exoscope. These patients experienced complication profiles similar to those that underwent the same treatments with the OM. The overall switch incidence rate from exoscope to $\mathrm{OM}$ during surgery was $5.8 \%$. Conclusions: The exoscope seems to be a safe alternative compared to an operative microscope for the most common brain and spinal procedures, with several advantages that have been reached, such as an easier simplicity of use and a better 3D vision and magnification of the surgical field. Moreover, it offers the opportunity of better interaction with other members of the surgical staff. All these points set the first step for subsequent and short-term changes in the field of neurosurgery and offer new educational possibilities for young neurosurgery and medical students.
\end{abstract}

Keywords: exoscope; high-definition 3D exoscope; augmented reality; intraoperative visualization; neurosurgery; brain tumor; virtual reality; spine; neuronavigation

\section{Introduction}

The surgical microscope has represented a basic tool in neurosurgery since the late 1960s, and it continues to be critically essential in the microsurgical treatment of brain and spine pathologies [1-8]. Advances in digital imaging, WiFi internet connections, screen technology and optics have led to the development of extracorporeal telescopes, also known as exoscopes, which represent valuable alternatives to traditional OMs for surgical magnification $[6,9,10]$. The use of the microscope requires that surgeons look directly through the surgical microscopic objective lenses to visualize the target area; however, it seems that this "face-to-machine" interface has been overcome due to the introduction and use of new digital 3-dimensional (3D) imaging exoscopes [2]. 
As widely reported in microsurgery and minimally invasive procedures, the pursuit of highly detailed images and techniques has been providing both valuable clinical results and patient satisfaction [11]. The technology of exoscopes has continuously changed over the years, and these devices are often updated in their software and hardware. Exoscopes have been the latest addition to the neurosurgeons' armamentarium, acting as a bridge between OMs and endoscopes [12]. The development of the 3D exoscope represents a marvel of technological innovation in modern surgical practice, which continues to renew itself year by year, from the first 3D High Definition (HD) visualization exoscope to the most recent 3D 4K exoscope. Furthermore, these modern exoscopes are embedded with light filters for 5-aminolevulinic acid (5-ALA) and indocyanine video-angiography, pneumatic arms, adjustable operative settings, multiscreen output, longer focus distance, and greater magnification powers $[13,14]$.

3D exoscopes are novel high-definition digital camera systems that are able to deliver intense light and magnification to the deepest areas of the surgical field, allowing the surgeon to see, through 3D glasses and a 3D monitor, critical neural and vascular structures as well as tissue differentiation with high magnification. A surgeon's position is not limited to the microscope' oculars, while freedom in movements during surgery, a higher comfort rate, a lower fatigue after longer procedures have been already reported in using an exoscope [9,15-18]. In neurosurgery, supports for various digitized information are essential for improving operative grades, as neurosurgeons could benefit from a new surgeon's eye that visualizes the operative field with integration of others medical information $[10,17]$.

Exoscopes, as well as other modern devices, require specific training, although the learning curve is very short when compared to other neurosurgical systems such as operative microscopes (OM) and endoscopes [5-7,19]. Siller et al. [5] reported no significant differences among patients who underwent surgery with OM or exoscope for lumbar posterior decompression (LPD) and anterior cervical discectomy and fusion (ACDF). Similarly, Muhammad et al. [7] reported results in cranial surgery comparable to the OM with better visual quality and greater comfort for the surgeon. The exoscope system is a safe and effective alternative or adjunct to the existing binocular OM for brain tumor, skull base surgery, aneurysm clipping and vascular microanastomosis, both cervical and lumbar complex spine surgery [5-8,10,20-25]. The exoscope provides the surgeon with a comfortable, high-resolution visualization without compromising surgical exposure and patient safety. The integrated features like the lock-on-target and waypoints together with the footswitch allow the surgeon to efficiently place the camera and to return to saved positions, even hands-free. All these functions in combination with the digital visualization are convenient and ergonomic compared to OM, even when the surgeon has to see into the situs using extreme angles. To date, several exoscopic systems are available for neurosurgical use. VITOM $^{\circledR}$ (Karl Storz, Tuttlingen, Germany), ORBEYE ${ }^{\mathrm{TM}}$ (Olympus, Tokyo, Japan), Modus

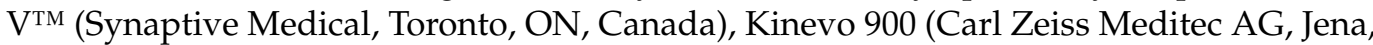
Germany), BrainPath ${ }^{\circledR}$ (Nico Corporation, Indianapolis, IN, US) and Aeos ${ }^{\circledR}$ (Aesculap, Tüttlingen, Germany) are the most commonly used, with different technical, software and hardware characteristics, but with the same goal [7,15,26-30]. As the exoscope will probably open a new era in the field of new tools and techniques in neurosurgery, as the OM did in the 1960s, this review aims to investigate about the use of the exoscope in preclinical and clinical neurosurgical settings, the most common neurosurgical procedures performed with the exoscope, as well as the impact of exoscope on surgical outcome and workflow, reporting operative complications, surgical procedures switched from exoscope to OM, and advantages and disadvantages compared to the microscope.

\section{Materials and Methods}

\subsection{Literature Search}

A Pubmed and Ovid EMBASE search was performed to identify papers that include surgical experiences with the exoscope in neurosurgery. PRISMA guidelines (Preferred Reporting Items for Systematic Reviews and Meta-analyses) were followed [31]. The 
key words "exoscope", "exoscopic visualization", "neurosurgery", "brain", "spine” and "cadaver lab" were used in both "AND" and "OR" combinations. The key words and the detailed search strategy are reported in Table 1.

Table 1. Search syntax.

\begin{tabular}{cc}
\hline PubMed Search Accessed on & Embase Search Accessed on \\
$\mathbf{5}$ July 2021 & 5 July 2021 \\
(108 Articles) & (106 Articles) \\
\hline (exoscope OR exoscopic visualization) AND & $\begin{array}{c}\text { ('exoscope' OR 'exoscopic visualization') AND } \\
\text { ('neurosurgery' OR 'brain' OR 'spine' OR } \\
\text { (neurosurgery OR brain OR spine OR cadaver } \\
\text { lab) }\end{array}$ \\
\hline
\end{tabular}

The inclusion criteria were the following: case series or case report reporting clinical data and neurosurgical intraoperative experiences with exoscope (both 2D and 3D visualization, as well 3D 4K definition) in brain and spine surgery as well as laboratory experiences in the field of neurosurgery. Exclusion criteria were the following: (1) studies published in languages other than English with no available English translations, (2) review articles, (3) studies with insufficient data, (4) studies not related with this topic.

\subsection{Data Collection}

From each study, we extracted the following data: (1) number of neurosurgical procedures performed using the exoscope divided by cerebral (tumor and vascular), spinal and peripheral nerve pathology as well as laboratory experiences; (2) exoscope manufacturer and/or model; (3) visualization mode setting; (4) operative complications and surgical procedures switched from the exoscope to OM; (5) advantages and disadvantages identified by authors (video image quality, surgical field, handling, surgical ergonomics, educational usefulness, depth perception, operative time and/or workflow, operative team involvement).

\subsection{Outcomes}

The primary objective of this review was to examine the most common neurosurgical procedures performed with the use of the exoscope and to identify surgical workflows, operative complications during the use of the exoscope and surgical procedures switched from the exoscope to the OM. The secondary objective was to report the most common advantages and disadvantages identified by authors (video image quality, surgical field, handling, surgical ergonomics, educational usefulness, depth perception, operative time and/or workflow, operative team involvement) to highlight strengths and weaknesses of this new technology.

\section{Results}

The database search yielded 208 articles. After the removal of duplicates, 108 articles were eligible for screening. A total of 86 articles met the selection criteria [3-10,13,15,16,19, 21,23,25-30,32-97]. The search flow diagram is shown in Figure 1. 


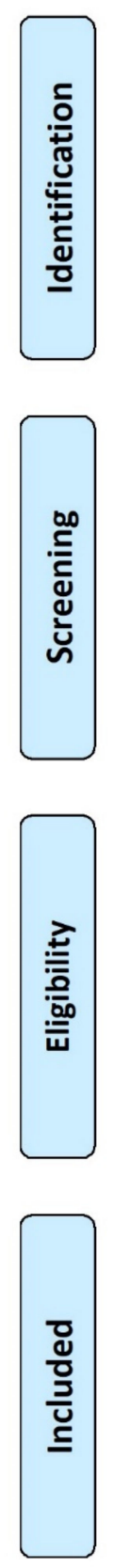

Records identified through
Pubmed database searching

$$
(n=107)
$$

Additional records identified through Ovid EMBASE data searching $(n=99)$

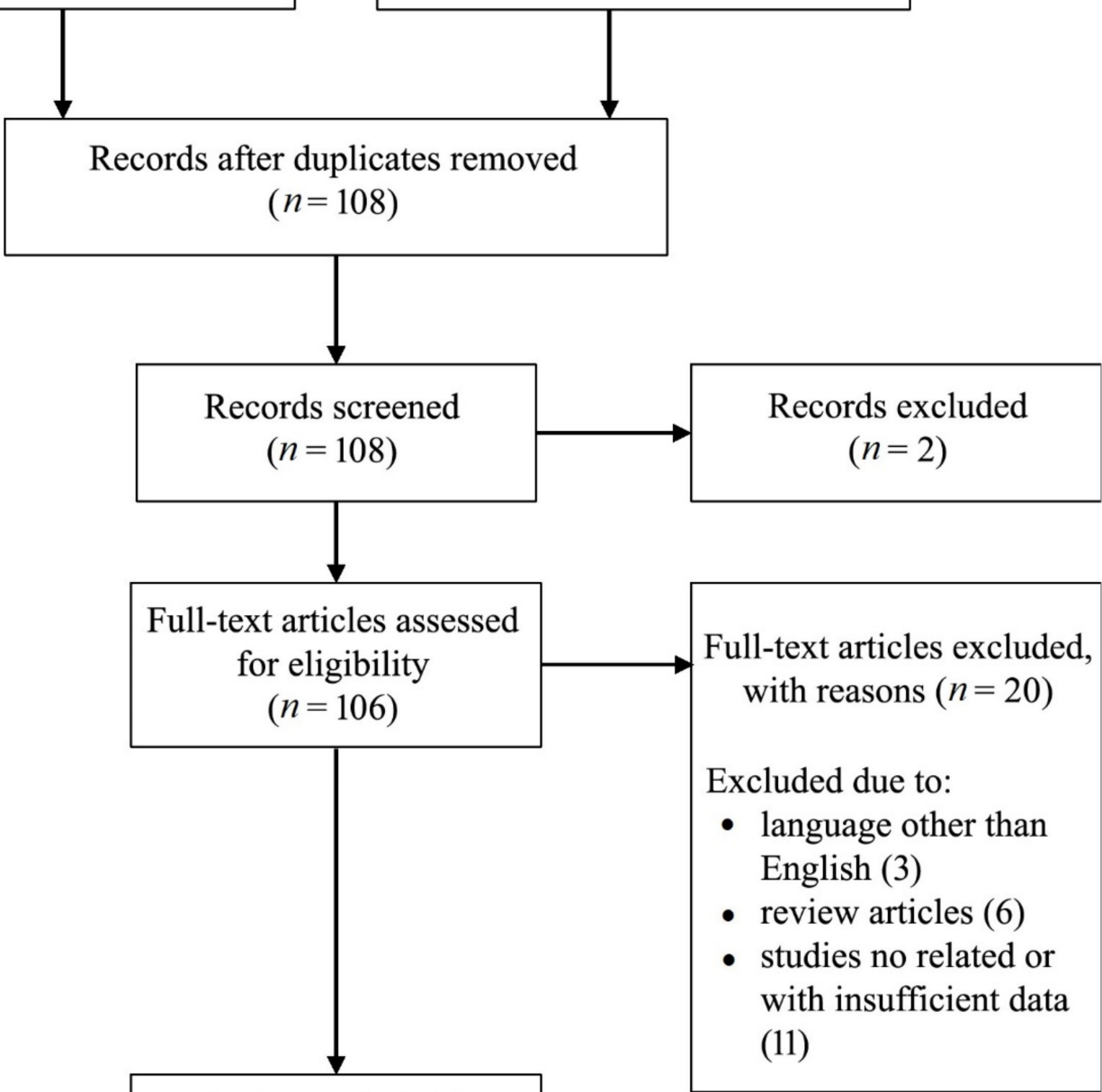

Figure 1. PRISMA flow diagram.

\subsection{Demographic and Number of Neurosurgical Procedures Performed Using Exoscope}

Studies included in our review are summarized in Table 2. A total of 86 articles and 1711 cases were included and analyzed in this review [3-10,13,15,16,19,21,23,25-30,32-97]. Among 86 papers included in the review 74 (86\%) were published in the last 5 years, showing an increasing interest in the use of the exoscope in the operating room in neurosurgery. Out of 1711 surgical procedures, 1534 (89.6\%) were performed on human beings in the operative room, whereas $177(10.9 \%)$ were performed in the laboratory on cadavers. A total of $1251(72.7 \%)$ were reported as brain surgeries, whereas $274(15.9 \%)$ and nine $(0.5 \%)$ were reported as spine and peripheral nerve surgeries, respectively. From this review of the literature, more than 311 gliomas, 171 brain metastasis and 97 meningioma and 244 pituitary adenomas were resected by using the exoscope. One hundred intracerebral hemorrhage (ICH) and 24 neurovascular conflicts were treated by using the exoscope. In spine surgery, 64 cervical disease, 189 lumbar pathologies and 12 spine tumors were reported. A total 
of 48 papers (55.8\%) reported their experience with a 3D HD exoscope, of which 15 used a $\mathrm{K} 4$ monitor, reporting the use of a $4 \mathrm{~K}$ HD exoscope in $17.4 \%$ of the papers. Excluding the 10 papers that report laboratory experiences, 21 papers $(27.6 \%)$ resulted in single case reports, 12 papers $(15.8 \%)$ were small series (with $\leq 5$ patients treated) and 43 papers $(56.6 \%)$ were clinical studies with a mean of 32.8 patients and a median of 18 patients. Most common exoscope manufacturers and/or models resulted VITOM ${ }^{\circledR}$ (Karl Storz, Tuttlingen, Germany) and ORBEYE ${ }^{\circledR}$ (Olympus, Tokyo, Japan), which were used and reported in $36 \%$ and in $23.3 \%$ of these review papers, respectively. Modus VTM (Synaptive Medical, Toronto, ON, Canada), BrainPath ${ }^{\circledR}$ (Nico Corporation, Indianapolis, IN, USA) and Kinevo 900 (Carl Zeiss Meditec AG, Jena, Germany) exoscopes were used in $9.3 \%, 7 \%$ and $3.5 \%$ of papers reported in this review. Tables 2 and 3 show all details about cranial and spine/peripheral nerve surgical procedures. Table 4 shows laboratory experiences with an exoscope.

Table 2. Summary of cranial studies included in the review.

\begin{tabular}{|c|c|c|c|c|c|c|}
\hline \multirow[t]{2}{*}{ Authors } & \multirow[t]{2}{*}{ Year } & \multicolumn{2}{|c|}{ Neurosurgical Procedures } & \multirow[b]{2}{*}{ Total } & \multirow{2}{*}{$\begin{array}{c}\text { Exoscope } \\
\text { Manufacturer } \\
\text { and/or Model }\end{array}$} & \multirow{2}{*}{$\begin{array}{l}\text { Visualization } \\
\text { Mode Setting }\end{array}$} \\
\hline & & Tumor $\left(n^{\circ}\right)$ & $\begin{array}{l}\text { Vascular and Others } \\
\text { Disease }\left(n^{\circ}\right)\end{array}$ & & & \\
\hline $\begin{array}{l}\text { Gildenberg \& } \\
\text { Labuz [32] }\end{array}$ & 1997 & $\begin{array}{c}\text { glioma (17), metastasis } \\
\text { (1) }\end{array}$ & - & 18 & $\mathrm{~N} / \mathrm{A}$ & - \\
\hline Mamelak et al. [34] & 2010 & $\begin{array}{l}\text { glioma (3), HMG (1), } \\
\text { meningioma (3), LGG } \\
(1) \text {, pituitary adenoma } \\
(1)\end{array}$ & $\begin{array}{l}\text { vagus nerve } \\
\text { stimulator }(1)\end{array}$ & 10 & $\begin{array}{l}\text { HDXO-SCOPE, } \\
\text { Karl Storz }\end{array}$ & HD 2D \\
\hline Mamelak et al. [36] & 2012 & germinoma (1) & - & 1 & VITOM $^{\circledR}$ & HD 2D \\
\hline Belloch et al. [37] & 2014 & $\begin{array}{c}\text { GBM (15), AA (2), } \\
\text { metastasis (3), LGG (3) }\end{array}$ & - & 23 & $\begin{array}{l}\text { HDXO-SCOPE, } \\
\text { Karl Storz }\end{array}$ & HD 2D \\
\hline Birch et al. [38] & 2014 & $\begin{array}{c}\text { pineocytoma (3), } \\
\text { germinoma (1), lipoma } \\
(1)\end{array}$ & - & 5 & $\begin{array}{l}\text { HDXO-SCOPE, } \\
\text { Karl Storz }\end{array}$ & HD 2D \\
\hline Piquer et al. [39] & 2014 & $\begin{array}{c}\text { GBM (23), AA (2), } \\
\text { metastasis (3), LGG (2) }\end{array}$ & - & 30 & VITOM $^{\circledR}$ & HD 2D \\
\hline Ritsma et al. [40] & 2014 & - & $\mathrm{ICH}(1)$ & 1 & Mi SPACE & HD 2D \\
\hline Parihar et al. [41] & 2016 & $\begin{array}{c}\text { meningioma (5), glioma } \\
\text { (4), HMG (1), metastasis } \\
(1), \text { schwannoma (3), } \\
\text { neurocytoma (1), } \\
\text { medulloblastoma (1), } \\
\text { craniopharyngioma (1) }\end{array}$ & $\begin{array}{l}\text { ICH (3), colloid cyst } \\
(1) \text {, arachnoid cyst (1), } \\
\text { abscess ( } 2), \text { trigeminal } \\
\text { neuralgia (1) }\end{array}$ & 25 & VITOM $^{\circledR}$ & HD 2D \\
\hline Scranton et al. [42] & 2016 & - & cavernoma (2) & 2 & $\mathrm{~N} / \mathrm{A}$ & HD 2D \\
\hline Bauer et al. [43] & 2017 & - & $\mathrm{ICH}(18)$ & 18 & BrainPath ${ }^{\circledR}$ & HD 2D \\
\hline Day [44] & 2017 & $\begin{array}{c}\text { GBM (15), AA (4), } \\
\text { ependymoma (2), } \\
\text { neurocytoma (1), LGG } \\
\text { (1), metastasis (20) }\end{array}$ & $\mathrm{ICH}(6)$ & 49 & BrainPath ${ }^{\circledR}$ & HD 2D \\
\hline Gonen et al. [45] & 2017 & $\begin{array}{c}\text { astrocytoma (56), } \\
\text { meningioma (40), } \\
\text { metastasis (33), } \\
\text { schwannoma (5), } \\
\text { epidermoid/dermoid } \\
\text { cyst (3), paraganglioma } \\
\text { (2), craniopharyngioma } \\
\text { (1), pituitary adenoma } \\
(1), \text { miscellaneous }(8)\end{array}$ & $\begin{array}{c}\text { aneurysms (7), AVM } \\
\text { (5), dAVF (1), ICH } \\
\text { (20), trigeminal } \\
\text { neuralgia (7), } \\
\text { hemifacial spasm (1), } \\
\text { arachnoid cysts ( } 2), \\
\text { Chiari I (1), infection } \\
\text { (3), colloid cyst (4) }\end{array}$ & 200 & ROVOT-m & HD 2D \\
\hline Jackson et al. [46] & 2017 & $\begin{array}{l}\text { GBM (3), AA (3), } \\
\text { metastasis (1), } \\
\text { lymphoma (2) }\end{array}$ & $\begin{array}{l}\text { demyelinating disease } \\
(2)\end{array}$ & 11 & VITOM $^{\circledR}$ & HD 2D \\
\hline
\end{tabular}


Table 2. Cont.

\begin{tabular}{|c|c|c|c|c|c|c|}
\hline \multirow[t]{2}{*}{ Authors } & \multirow[t]{2}{*}{ Year } & \multicolumn{2}{|c|}{ Neurosurgical Procedures } & \multirow[b]{2}{*}{ Total } & \multirow{2}{*}{$\begin{array}{l}\text { Exoscope } \\
\text { Manufacturer } \\
\text { and/or Model }\end{array}$} & \multirow{2}{*}{$\begin{array}{l}\text { Visualization } \\
\text { Mode Setting }\end{array}$} \\
\hline & & Tumor $\left(n^{\circ}\right)$ & $\begin{array}{l}\text { Vascular and Others } \\
\text { Disease }\left(n^{\circ}\right)\end{array}$ & & & \\
\hline Krishnan et al. [47] & 2017 & - & $\begin{array}{c}\text { anastomosis (3), AVF } \\
(1), \mathrm{ICH}(1)\end{array}$ & 3 & VITOM $^{\circledR}$ & HD 2D \\
\hline Labib et al. [48] & 2017 & - & ICH (39) & 39 & Mi SPACE & HD 2D \\
\hline Nagata et al. [50] & 2017 & - & hemifacial spasm (2) & 2 & ORBEYE $^{\circledR}$ & HD 2D \\
\hline Oertel \& Burkhardt [51] & 2017 & $\begin{array}{l}\text { metastasis (3), } \\
\text { lymphoma (1) }\end{array}$ & $\begin{array}{l}\text { trigeminal neuralgia } \\
\text { (1) }\end{array}$ & 5 & VITOM $^{\circledR}$ & HD 3D \\
\hline Rossini et al. [52] & 2017 & meningioma (1) & - & 1 & VITOM $^{\circledR}$ & HD 3D \\
\hline $\begin{array}{c}\text { Weiner \& } \\
\text { Placantonakis [53] }\end{array}$ & 2017 & JPA (1) & - & 1 & VITOM $^{\circledR}$ & HD 3D \\
\hline Beez et al. [54] & 2018 & astrocytoma (2) & $\begin{array}{l}\text { myelomeningocele } \\
\text { closure (1) }\end{array}$ & 3 & VITOM $^{\circledR}$ & HD 3D \\
\hline Gassie et al. [55] & 2018 & $\begin{array}{l}\text { GBM (24), AA (6), } \\
\text { metastasis (14), } \\
\text { lymphoma (2) }\end{array}$ & $\begin{array}{c}\text { cavernoma (2), } \\
\text { demyelinating disease } \\
(2)\end{array}$ & 50 & VITOM $^{\circledR}$ & HD 2D \\
\hline Griessenauer et al. [56] & 2018 & - & $\mathrm{ICH}(5)$ & 5 & BrainPath $^{\circledR}$ & HD 2D \\
\hline Iyer \& Chaichana [57] & 2018 & GBM (11), AA (3) & - & 14 & VITOM $^{\circledR}$ & HD 2D \\
\hline Khalessi et al. [58] & 2018 & $\begin{array}{l}\text { meningioma }(1) \text {, glioma } \\
\text { (1) }\end{array}$ & $\begin{array}{c}\text { clipping (4), } \\
\text { cavernoma (3), AVM } \\
\text { (2), endarterectomy } \\
\text { (1), CSDH (1), cyst (1), } \\
\text { Chiari I (1) }\end{array}$ & 17 & ORBEYE ${ }^{\circledR}$ & HD 3D \\
\hline Klinger et al. [59] & 2018 & - & aneurysm (1) & 1 & Modus VTM & HD 3D \\
\hline Mampre et al. [60] & 2018 & $\begin{array}{c}\text { metastasis (11), HMG } \\
(2)\end{array}$ & cavernoma (2) & 15 & VITOM $^{\circledR}$ & HD 2D \\
\hline Sindelar et al. [8] & 2018 & - & $\mathrm{ICH}(1)$ & 1 & BrainPath $^{\circledR}$ & HD 2D \\
\hline Takahashi et al. [61] & 2018 & $\begin{array}{c}\text { meningioma (5), } \\
\text { pituitary adenoma (1), } \\
\text { GBM (1), HMG (2), } \\
\text { metastasis (1), } \\
\text { craniopharyngioma (1) }\end{array}$ & $\begin{array}{l}\text { Moyamoya disease } \\
(2), \text { congenital dermal } \\
\text { sinus (1) }\end{array}$ & 14 & ORBEYE ${ }^{\circledR}$ & HD 2D \\
\hline Akbari et al. [62] & 2019 & $\begin{array}{c}\text { metastasis (4), GBM (3), } \\
\text { LGG (2), AA (1) }\end{array}$ & - & 10 & VITOM $^{\circledR}$ & HD 2D \\
\hline Angileri et al. [4] & 2019 & - & $\begin{array}{c}\text { cavernoma + HMG } \\
(1)\end{array}$ & 1 & VITOM $^{\circledR}$ & HD 3D \\
\hline Bakhsheshian et al. [63] & 2019 & metastasis (25) & - & 25 & BrainPath $^{\circledR}$ & HD 3D \\
\hline Garneau et al. [16] & 2019 & schwannoma (4) & $\begin{array}{l}\text { temporal lobe } \\
\text { encephalocele (2) }\end{array}$ & 6 & Modus V ${ }^{\mathrm{TM}}$ & HD 2D \\
\hline $\begin{array}{l}\text { Li Ching Ng \& Di } \\
\text { Ieva [66] }\end{array}$ & 2019 & - & MVD (1) & 1 & VITOM $^{\circledR}$ & HD 3D \\
\hline Muhammad et al. [7] & 2019 & $\begin{array}{l}\text { schwannoma (1), } \\
\text { meningioma (3) }\end{array}$ & - & 4 & Modus $V^{\mathrm{TM}}$ & HD 3D \\
\hline Murai et al. [67] & 2019 & $\begin{array}{c}\text { meningioma (3), } \\
\text { schwannoma (3), } \\
\text { pituitary adenoma (1), } \\
\text { GBM (1) }\end{array}$ & $\begin{array}{l}\text { clipping (3), bypass } \\
\text { (2), carotid } \\
\text { endarterectomy (2), } \\
\text { ICH (3) }\end{array}$ & 18 & ORBEYE ${ }^{\circledR}$ & $3 \mathrm{D} 4 \mathrm{~K}$ \\
\hline Nossek et al. [68] & 2019 & - & bypass (5) & 5 & $\mathrm{ORBEYE}^{\circledR}$ & $3 \mathrm{D} 4 \mathrm{~K}$ \\
\hline Smith et al. [69] & 2019 & - & skull base (11) & 11 & $\begin{array}{c}\text { ORBEYE }^{\circledR}(10), \\
\operatorname{VITOM}^{\circledR}(1)\end{array}$ & HD 3D \\
\hline Ahmad et al. [9] & 2020 & - & $\begin{array}{l}\text { microvascular } \\
\text { anastomosis (12) }\end{array}$ & 12 & ORBEYE ${ }^{\circledR}$ & HD 3D \\
\hline
\end{tabular}


Table 2. Cont.

\begin{tabular}{|c|c|c|c|c|c|c|}
\hline \multirow[t]{2}{*}{ Authors } & \multirow[t]{2}{*}{ Year } & \multicolumn{2}{|c|}{ Neurosurgical Procedures } & \multirow[b]{2}{*}{ Total } & \multirow{2}{*}{$\begin{array}{c}\text { Exoscope } \\
\text { Manufacturer } \\
\text { and/or Model }\end{array}$} & \multirow[b]{2}{*}{$\begin{array}{l}\text { Visualization } \\
\text { Mode Setting }\end{array}$} \\
\hline & & Tumor $\left(n^{\circ}\right)$ & $\begin{array}{l}\text { Vascular and Others } \\
\text { Disease }\left(n^{\circ}\right)\end{array}$ & & & \\
\hline Baron et al. [10] & 2020 & GBM (28) & - & 28 & Modus $\mathrm{V}^{\mathrm{TM}}$ & HD 3D \\
\hline Burkhardt et al. [71] & 2020 & $\begin{array}{c}\text { metastasis (3), LGG (1), } \\
\text { AA (1), GBM (2), } \\
\text { meningioma (1), } \\
\text { subependymoma (1), } \\
\text { lymphoma (1) }\end{array}$ & $\begin{array}{c}\text { cavernoma (1), ICH } \\
(1) \text {, aneurysm (2), CSF } \\
\text { leak (1), trigeminal } \\
\text { neuralgia (1) }\end{array}$ & 16 & VITOM $^{\circledR}$ & HD 3D \\
\hline Chakravarthi et al. [72] & 2020 & hypothalamic mass (1) & - & 1 & Kinevo 900 & HD 3D \\
\hline Chen et al. [73] & 2020 & schwannoma (39) & - & 39 & VITOM $^{\circledR}$ & HD 2D \\
\hline Doglietto et al. [76] & 2020 & GBM (1) & - & 1 & ORBEYE ${ }^{\circledR}$ & $3 \mathrm{D} 4 \mathrm{~K}$ \\
\hline Eichberg et al. [30] & 2020 & $\begin{array}{l}\text { GBM (13), metastasis } \\
(19) \text {, glioma (8) }\end{array}$ & $\begin{array}{l}\text { cavernoma }(7), \text { colloid } \\
\text { cyst }(4), \text { other }(5)\end{array}$ & 56 & BrainPath $^{\circledR}$ & HD 2D \\
\hline Fuse et al. [3] & 2020 & meningioma (1) & - & 1 & VITOM $^{\circledR}$ & HD 2D \\
\hline Garneau et al. [77] & 2020 & - & $\begin{array}{c}\text { temporal lobe } \\
\text { encephalocele (1) }\end{array}$ & 1 & Modus VTM & HD 3D \\
\hline Khatri et al. [78] & 2020 & craniopharyngioma (1) & - & 1 & $\mathrm{~N} / \mathrm{A}$ & - \\
\hline Kleshchova et al. [79] & 2020 & endodermal cyst (1) & - & 1 & $\mathrm{~N} / \mathrm{A}$ & - \\
\hline Ligas et al. [80] & 2020 & - & hemifacial spasm (1) & 1 & $\mathrm{~N} / \mathrm{A}$ & - \\
\hline Lin et al. [81] & 2020 & meningioma (4) & - & 4 & VITOM $^{\circledR}$ & HD 3D \\
\hline Patel et al. [84] & 2020 & - & bypass (1) & 1 & $\mathrm{~N} / \mathrm{A}$ & - \\
\hline Roethe et al. [85] & 2020 & $\begin{array}{c}\text { GBM (9), meningioma } \\
\text { (6), LGG (4), metastasis } \\
\text { (3), AA (3) }\end{array}$ & $\begin{array}{l}\text { cavernoma (1), } \\
\text { trigeminal neuralgia } \\
(1), \text { CSF fistula }(1)\end{array}$ & 28 & Kinevo 900 & $3 \mathrm{D} 4 \mathrm{~K}$ \\
\hline Silverstein et al. [87] & 2020 & - & aneurysm (1) & 1 & ORBEYE $^{\circledR}$ & HD 3D \\
\hline Amoo et al. [90] & 2021 & $\begin{array}{c}\text { metastasis (5), } \\
\text { meningioma (4), GBM } \\
(5), \text { schwannoma (1), } \\
\text { craniopharyngioma (1) }\end{array}$ & $\begin{array}{l}\text { AVM (1), hemifacial } \\
\text { spasm (1) }\end{array}$ & 18 & ORBEYE ${ }^{\circledR}$ & $3 \mathrm{D} 4 \mathrm{~K}$ \\
\hline $\begin{array}{c}\text { Marenco-Hillembrand } \\
\text { et al. [92] }\end{array}$ & 2021 & $\begin{array}{c}\text { metastasis (8), LGG (4), } \\
\text { GBM (3) }\end{array}$ & - & 15 & $\mathrm{~N} / \mathrm{A}$ & - \\
\hline Muscas et al. [27] & 2021 & $\begin{array}{c}\text { meningioma (4), cranial } \\
\text { nerve tumors (2), } \\
\text { glioma (3), choroid } \\
\text { plexus papilloma (1) }\end{array}$ & $\begin{array}{l}\text { aneurysm (1), colloid } \\
\text { cyst (1), } \\
\text { neurovascular conflict } \\
(1), \text { ethmoidal fistula } \\
(1)\end{array}$ & 14 & ORBEYE ${ }^{\circledR}$ & $3 \mathrm{D} 4 \mathrm{~K}$ \\
\hline Muto et al. [93] & 2021 & metastasis (5) & - & 5 & VS3 Iridium & HD 3D \\
\hline Rennert et al. [94] & 2021 & GBM (1) & $\mathrm{ICH}(1)$ & 2 & VITOM $^{\circledR}$ & HD 3D \\
\hline Rösler et al. [26] & 2021 & $\begin{array}{c}\text { GBM (6), pituitary } \\
\text { adenoma (1), } \\
\text { meningioma (1), } \\
\text { craniopharyngioma (1), } \\
\text { LGG (4), lymphoma (1), } \\
\text { metastasis (3), HMG (1), } \\
\text { hemangioma (1) }\end{array}$ & $\begin{array}{l}\mathrm{ICH}(1), \text { epilepsy (6), } \\
\text { trigeminal neuralgia } \\
\text { (1) }\end{array}$ & 27 & ORBEYE ${ }^{\circledR}$ & $3 \mathrm{D} 4 \mathrm{~K}$ \\
\hline Rotermund et al. [95] & 2021 & $\begin{array}{l}\text { pituitary adenoma (239), } \\
\text { craniopharyngioma (12), } \\
\text { meningioma (7), } \\
\text { chordoma (4), } \\
\text { metastasis (2) }\end{array}$ & other (32) & 296 & ORBEYE ${ }^{\circledR}$ & $3 \mathrm{D} 4 \mathrm{~K}$ \\
\hline Shimizu et al. [96] & 2021 & $\begin{array}{l}\text { meningioma (5), } \\
\text { schwannoma (4) }\end{array}$ & $\begin{array}{l}\text { trigeminal neuralgia } \\
(2), \text { hemifacial spasm } \\
\text { (3) }\end{array}$ & 14 & ORBEYE ${ }^{\circledR}$ & $3 \mathrm{D} 4 \mathrm{~K}$ \\
\hline
\end{tabular}


Table 2. Cont.

\begin{tabular}{|c|c|c|c|c|c|c|}
\hline \multirow[t]{2}{*}{ Authors } & \multirow[t]{2}{*}{ Year } & \multicolumn{2}{|c|}{ Neurosurgical Procedures } & \multirow[b]{2}{*}{ Total } & \multirow{2}{*}{$\begin{array}{c}\text { Exoscope } \\
\text { Manufacturer } \\
\text { and/or Model }\end{array}$} & \multirow[b]{2}{*}{$\begin{array}{l}\text { Visualization } \\
\text { Mode Setting }\end{array}$} \\
\hline & & Tumor $\left(n^{\circ}\right)$ & $\begin{array}{l}\text { Vascular and Others } \\
\text { Disease }\left(n^{\circ}\right)\end{array}$ & & & \\
\hline Strickland et al. [97] & 2021 & - & $\operatorname{AVM}(1)$ & 1 & $\mathrm{~N} / \mathrm{A}$ & - \\
\hline Wali et al. [21] & 2021 & - & aneurysm (1) & 1 & ORBEYE $^{\circledR}$ & $3 \mathrm{D} 4 \mathrm{~K}$ \\
\hline Yoon et al. [25] & 2021 & $\begin{array}{c}\text { metastasis (3), } \\
\text { meningioma (3), GBM } \\
\text { (4), HMG (1) }\end{array}$ & - & 11 & $\begin{array}{l}\text { VOMS-100 (5), } \\
\text { VITOM }^{\circledR}(6)\end{array}$ & $3 \mathrm{D} 4 \mathrm{~K}$ \\
\hline
\end{tabular}

2d, 2 dimensional; 3D 4K, 3 dimensional 4K high-definition; AA, anaplastic astrocytoma; AVM, arteriovenous malformation; CSF, cerebrospinal fluid; CSDH, chronic subdural hematoma; dAVF, arteriovenous fistula; GBM glioblastoma multiforme; HD, high definition; HMG, hemangioblastomas; ICH, intracerebral hemorrhage; N/A, not available; JPA, juvenile pilocytic astrocytoma; LGG, low-grade glioma; MVD, microvascular decompression.

Table 3. Summary of spine/peripheral nerve studies included in the review.

\begin{tabular}{|c|c|c|c|c|c|c|}
\hline \multirow[t]{2}{*}{ Authors } & \multirow[t]{2}{*}{ Year } & \multicolumn{2}{|c|}{ Neurosurgical Procedures } & \multirow{2}{*}{ Total } & \multirow{2}{*}{$\begin{array}{c}\text { Exoscope } \\
\text { Manufacturer } \\
\text { and/or Model }\end{array}$} & \multirow{2}{*}{$\begin{array}{l}\text { Visualization } \\
\text { Mode Setting }\end{array}$} \\
\hline & & Spine $\left(n^{\circ}\right)$ & Peripheral $\left(\mathrm{n}^{\circ}\right)$ & & & \\
\hline Mamelak et al. [34] & 2010 & $\begin{array}{c}\text { ACDF (2), epidural abscess (1), } \\
\text { lumbar discectomy (3) }\end{array}$ & - & 6 & $\begin{array}{l}\text { HDXO-SCOPE, } \\
\text { Karl Storz }\end{array}$ & HD 2D \\
\hline Shirzadi et al. [19] & 2012 & LPD (11), TLIF (13) & - & 24 & VITOM $^{\circledR}$ & HD 2D \\
\hline Parihar et al. [41] & 2016 & $\begin{array}{l}\text { neurofibroma (4), meningioma } \\
\text { (1), ACDF (4), corpectomy (2), } \\
\text { tuberculosis (1), lumbar } \\
\text { discectomy (2) }\end{array}$ & - & 14 & VITOM $^{\circledR}$ & HD 2D \\
\hline Krishnan et al. [47] & 2017 & $\begin{array}{l}\operatorname{LPD}(7) \text {, cervical } \\
\text { foraminotomy (2), ACDF (1) }\end{array}$ & $\begin{array}{l}\text { schwannoma (2), } \\
\text { microneurorrhaphy (1) }\end{array}$ & 13 & VITOM $^{\circledR}$ & HD 2D \\
\hline Oertel \& Burkhardt [51] & 2017 & $\begin{array}{c}\text { ACDF (2), cervical } \\
\text { laminectomies (2), TLIF (2), } \\
\text { extradural tumor (1), LPD (1), } \\
\text { lumbar discectomy (3) }\end{array}$ & - & 11 & VITOM $^{\circledR}$ & HD 3D \\
\hline Khalessi et al. [58] & 2018 & ACDF (1), disc herniation (2) & - & 3 & ORBEYE ${ }^{\circledR}$ & HD 3D \\
\hline De Divitiis et al. [6] & 2019 & tumor (5) & - & 5 & VITOM $^{\circledR}$ & HD 3D \\
\hline Kwan et al. [65] & 2019 & $\begin{array}{l}\text { ACDF (4), cervical corpectomy } \\
\text { (1), cervical laminectomies (3), } \\
\text { LPD (2) }\end{array}$ & - & 10 & ORBEYE ${ }^{\circledR}$ & HD 3D \\
\hline Muhammad et al. [7] & 2019 & $\begin{array}{l}\text { CPD (1), ACDF (1), disc } \\
\text { herniation (2) }\end{array}$ & - & 4 & Modus $\mathrm{V}^{\mathrm{TM}}$ & HD 3D \\
\hline Murai et al. [67] & 2019 & $\operatorname{LDP}(3)$ & neurolysis (1) & 4 & ORBEYE ${ }^{\circledR}$ & $3 \mathrm{D} 4 \mathrm{~K}$ \\
\hline Ariffin et al. [15] & 2020 & $\begin{array}{l}\text { decompression (18), } \\
\text { discectomy (17), TLIF (28), } \\
\text { OLIF (6) }\end{array}$ & - & 69 & Kinevo 900 & $3 \mathrm{D} 4 \mathrm{~K}$ \\
\hline Barbagallo \& Certo [70] & 2020 & $\operatorname{ACDF}(2)$ & - & 2 & VITOM $^{\circledR}$ & HD 3D \\
\hline Burkhardt et al. [71] & 2020 & $\begin{array}{c}\text { ACDF (4), cervical } \\
\text { laminectomies (1), metastasis } \\
(1) \text {, lumbar decompression (4), } \\
\text { TLIF (1), disc herniation (5), } \\
\text { dAVF (1), angiolipoma (1) }\end{array}$ & - & 18 & VITOM $^{\circledR}$ & HD 3D \\
\hline D'Ercole et al. [75] & 2020 & ALIF (9) & - & 9 & VITOM $^{\circledR}$ & HD 3D \\
\hline Oren et al. [82] & 2020 & disc herniation (1) & - & 1 & ORBEYE ${ }^{\circledR}$ & $3 \mathrm{D} 4 \mathrm{~K}$ \\
\hline Roethe et al. [85] & 2020 & LPD (1) & - & 1 & Kinevo 900 & $3 \mathrm{D} 4 \mathrm{~K}$ \\
\hline Siller et al. [5] & 2020 & $\operatorname{LDP}(40), \operatorname{ACDF}(20)$ & - & 60 & VITOM $^{\circledR}$ & HD 3D \\
\hline Teo et al. [29] & 2020 & $\begin{array}{l}\text { fracture (2), meningioma (1), } \\
\text { disc herniation (5) }\end{array}$ & - & 8 & Modus V VM & HD 3D \\
\hline
\end{tabular}


Table 3. Cont.

\begin{tabular}{|c|c|c|c|c|c|c|}
\hline \multirow[t]{2}{*}{ Authors } & \multirow[t]{2}{*}{ Year } & \multicolumn{2}{|c|}{ Neurosurgical Procedures } & \multirow{2}{*}{ Total } & \multirow{2}{*}{$\begin{array}{c}\text { Exoscope } \\
\text { Manufacturer } \\
\text { and/or Model }\end{array}$} & \multirow{2}{*}{$\begin{array}{l}\text { Visualization } \\
\text { Mode Setting }\end{array}$} \\
\hline & & Spine $\left(\mathbf{n}^{\circ}\right)$ & Peripheral $\left(\mathrm{n}^{\circ}\right)$ & & & \\
\hline Vetrano et al. [88] & 2020 & - & schwannoma (2) & 2 & ORBEYE ${ }^{\circledR}$ & $3 \mathrm{D} 4 \mathrm{~K}$ \\
\hline Visocchi et al. [89] & 2020 & CVJ pathologies (6) & - & 6 & $\begin{array}{l}\text { VITOM }^{\circledR}(3), \\
\text { ORBEYE }^{\circledR}(3)\end{array}$ & $3 \mathrm{D} 4 \mathrm{~K}$ \\
\hline Kim et al. [91] & 2021 & disc herniation (1) & - & 1 & $\mathrm{~N} / \mathrm{A}$ & - \\
\hline Rösler et al. [26] & 2021 & $\begin{array}{l}\text { ACDF (1), metastasis (1), } \\
\text { tumor (1), schwannoma (2), } \\
\text { LPD (4) }\end{array}$ & $\begin{array}{c}\text { schwannoma (2), } \\
\text { peripheral nerve (1) }\end{array}$ & 12 & ORBEYE ${ }^{\circledR}$ & $3 \mathrm{D} 4 \mathrm{~K}$ \\
\hline
\end{tabular}

3D 4K, 3 dimensional 4K high-definition; ACDF, anterior cervical discectomy and fusion; ALIF, anterior lumbar interbody fusion; CPD, cervical posterior decompression; CVJ, craniovertebral junction; HD, high definition; LPD, lumbar posterior decompression; OLIF, oblique lateral interbody fusion; TLIF, transforaminal lumbar interbody fusion.

Table 4. Summary of laboratory studies included in the review.

\begin{tabular}{|c|c|c|c|c|c|}
\hline Authors & Year & $\begin{array}{l}\text { Laboratory Neurosurgical } \\
\text { Procedures }\left(n^{\circ}\right)\end{array}$ & Total & $\begin{array}{c}\text { Exoscope } \\
\text { Manufacturer } \\
\text { and/or Model }\end{array}$ & $\begin{array}{l}\text { Visualization } \\
\text { Mode Setting }\end{array}$ \\
\hline Mamelak et al. [33] & 2008 & craniotomy (4) & 4 & $\begin{array}{l}\text { HDXO-SCOPE, } \\
\text { Karl Storz }\end{array}$ & HD 2D \\
\hline Di Ieva et al. [35] & 2012 & suboccipital approach (20) & 20 & VITOM $^{\circledR}$ & HD 2D \\
\hline Moisi et al. [49] & 2017 & craniotomy (6) & 6 & Modus $\mathrm{V}^{\mathrm{TM}}$ & HD 2D \\
\hline Sack et al. [13] & 2018 & craniotomy (5) & 5 & ORBEYE $^{\circledR}$ & HD 3D \\
\hline Herlan et al. [64] & 2019 & pterional approach (6) & 6 & FA Aesculap & HD 3D \\
\hline Crosetti et al. [74] & 2020 & dissection (4) & 4 & VITOM $^{\circledR}$ & HD 3D \\
\hline Hafez et al. [23] & 2020 & bypass anastomosis (100) & 100 & VITOM $^{\circledR}$ & HD 3D \\
\hline Pafitanis et al. [83] & 2020 & $\begin{array}{l}\text { micro sutures (10), } \\
\text { anastomoses (5) }\end{array}$ & 15 & Modus $\mathrm{V}^{\mathrm{TM}}$ & HD 3D \\
\hline Rubini et al. [86] & 2020 & skull base (12) & 12 & VITOM $^{\circledR}$ & HD 3D \\
\hline Hafez et al. [28] & 2021 & bypass (5) & 5 & AEOS & 3D 4K \\
\hline
\end{tabular}

3D 4K, 3 dimensional 4K high-definition; HD, high definition.

\subsection{Evaluation of Exoscopic Surgical Procedures}

Excluding case reports and considering clinical series reporting surgical complications, 40 studies and 1328 patients were assessed, reporting an overall surgical complication rate of $2.6 \%$ during the use of the exoscope. These patients experienced complication profiles similar to those that underwent the same treatments with the OM. Similarly, 21 clinical series with a total of 891 patients reported an overall switch incidence rate from exoscope to OM during surgery of $5.8 \%$ (52 cases). A total of 30 articles reported a qualitative comparison between the exoscope and the OM, while a total of 12 papers reported a quantitative, concrete and prospective comparison between one or more common features of the exoscope with the OM. The video image quality, 3D visualization and surgical filed with exoscopes were rated superior to similar to those of OMs in all papers. The comfort level of surgeon's posture during surgery, the educational usefulness, and the operative team involvement with the exoscope were assessed as superior compared to OM. Otherwise, depth perception was rated to be similar or inferior to the OM. Workflow and operative time were evaluated as equal or slightly higher than those of OMs. Table 5 shows all details. 
Table 5. Summary of evaluation exoscopic surgical procedures studies.

\begin{tabular}{|c|c|c|c|c|c|c|c|c|c|c|c|c|}
\hline Authors & Year & $\begin{array}{c}\text { Patients } \\
\left(\mathbf{n}^{\circ}\right)\end{array}$ & $\begin{array}{c}\text { Operative } \\
\text { complications } \\
(\%)\end{array}$ & $\begin{array}{l}\text { Surgical Procedures } \\
\text { Switched from } \\
\text { Exoscope to OM (\%) }\end{array}$ & $\begin{array}{l}\text { Video Image } \\
\text { Quality }\end{array}$ & Surgical Field & Handling & $\begin{array}{c}\text { Surgical } \\
\text { Ergonomics }\end{array}$ & $\begin{array}{l}\text { Educational } \\
\text { Usefulness }\end{array}$ & $\begin{array}{c}\text { Depth } \\
\text { Perception }\end{array}$ & $\begin{array}{c}\text { Operative } \\
\text { Time and/or } \\
\text { Workflow }\end{array}$ & $\begin{array}{c}\text { Operative } \\
\text { Team } \\
\text { Involvemen }\end{array}$ \\
\hline Mamelak et al. [34] & 2010 & 16 & 0 & $\mathrm{Nd}$ & + & + & & + & + & & & + \\
\hline Shirzadi et al. [19] & 2012 & 24 & 0 & $\mathrm{Nd}$ & $=$ & & & & & & $=$ & \\
\hline Belloch et al. [37] & 2014 & 23 & 0 & $\mathrm{Nd}$ & & & + & + & & & & \\
\hline Birch et al. [38] & 2014 & 5 & 20 & $\mathrm{Nd}$ & & $=$ & & & & & $=$ & \\
\hline Piquer et al. [39] & 2014 & 30 & 0 & $\mathrm{Nd}$ & + & + & & + & & & & \\
\hline Parihar et al. [41] & 2016 & 39 & 0 & 0 & $=$ & & & + & & - & & \\
\hline Day [44] & 2017 & 49 & 8.2 & $\mathrm{Nd}$ & & + & + & & & & & \\
\hline Gonen et al. [45] & 2017 & 200 & 0 & 0 & + & + & & & & & & + \\
\hline Jackson et al. [46] & 2017 & 11 & 0 & 0 & + & + & & & & & & \\
\hline Krishnan et al. [47] & 2017 & 18 & $\mathrm{Nd}$ & 0 & + & + & + & & & - & - & \\
\hline Labib et al. [48] & 2017 & 39 & 7.7 & $\mathrm{Nd}$ & + & + & & & & & & \\
\hline Oertel \& Burkhardt [51] & 2017 & 16 & 0 & 0 & $=$ & $=$ & + & + & & $=$ & & + \\
\hline Iyer \& Chaichana [57] & 2018 & 14 & 0 & $\mathrm{Nd}$ & + & + & + & & & & & \\
\hline Khalessi et al. [58] & 2018 & 18 & 0 & $\mathrm{Nd}$ & + & + & + & + & & $=$ & & + \\
\hline Bakhsheshian et al. [63] & 2019 & 25 & 4 & 0 & + & + & & & & & & . \\
\hline Garneau et al. [16] & 2019 & 6 & 0 & 0 & + & + & & + & & - & - & + \\
\hline Herlan et al. [64] & 2019 & 6 & NA & $\mathrm{Nd}$ & + & $=$ & & + & & $=$ & & \\
\hline Kwan et al. [65] & 2019 & 10 & 0 & $\mathrm{Nd}$ & + & + & + & + & + & & & + \\
\hline Muhammad et al. [7] & 2019 & 8 & 0 & 0 & + & + & & + & & - & & \\
\hline Murai et al. [67] & 2019 & 22 & 0 & $18.2 \%$ & + & & & + & + & & & \\
\hline Smith et al. [69] & 2019 & 11 & 0 & $36.4 \%$ & $=$ & $=$ & & + & + & & & + \\
\hline Ahmad et al. [9] & 2020 & 22 & 0 & $\mathrm{Nd}$ & + & + & + & + & & & $=$ & \\
\hline Ariffin et al. [15] & 2020 & 69 & 5.8 & $\mathrm{Nd}$ & + & + & + & + & + & + & & + \\
\hline Baron et al. [10] & 2020 & 28 & 3.6 & $\mathrm{Nd}$ & + & + & & & & & & \\
\hline Burkhardt et al. [71] & 2020 & 34 & $\mathrm{Nd}$ & $17.6 \%$ & $=/+$ & $=/+$ & & & & - & & \\
\hline Chen et al. [73] & 2020 & 39 & 30.8 & $\mathrm{Nd}$ & & $=$ & & + & & & $=$ & + \\
\hline Eichberg et al. [30] & 2020 & 56 & 8.9 & $\mathrm{Nd}$ & $=$ & $=$ & & $=$ & & & & \\
\hline
\end{tabular}


Table 5. Cont.

\begin{tabular}{|c|c|c|c|c|c|c|c|c|c|c|c|c|}
\hline Authors & Year & $\begin{array}{c}\text { Patients } \\
\left(\mathbf{n}^{\circ}\right)\end{array}$ & $\begin{array}{c}\text { Operative } \\
\text { complications } \\
(\%)\end{array}$ & $\begin{array}{l}\text { Surgical Procedures } \\
\text { Switched from } \\
\text { Exoscope to OM (\%) }\end{array}$ & $\begin{array}{l}\text { Video Image } \\
\text { Quality }\end{array}$ & Surgical Field & Handling & $\begin{array}{c}\text { Surgical } \\
\text { Ergonomics }\end{array}$ & $\begin{array}{l}\text { Educational } \\
\text { Usefulness }\end{array}$ & $\begin{array}{c}\text { Depth } \\
\text { Perception }\end{array}$ & $\begin{array}{l}\text { Operative } \\
\text { Time and/or } \\
\text { Workflow }\end{array}$ & $\begin{array}{c}\text { Operative } \\
\text { Team } \\
\text { Involvement }\end{array}$ \\
\hline Hafez et al. [23] & 2020 & 100 & NA & $\mathrm{Nd}$ & $=$ & + & & & + & & & \\
\hline Pafitanis et al. [83] & 2020 & 15 & NA & $\mathrm{Nd}$ & $=$ & $=$ & & & & & & \\
\hline Roethe et al. [85] & 2020 & 29 & 6.9 & $3.4 \%$ & - & $=$ & $=$ & + & & & $=$ & \\
\hline Siller et al. [5] & 2020 & 60 & 0 & 0 & - & $=$ & $=$ & + & + & - & $=$ & + \\
\hline Teo et al. [29] & 2020 & 8 & 0 & $\mathrm{Nd}$ & $=/+$ & $=/+$ & & & $=/+$ & & & \\
\hline Visocchi et al. [89] & 2020 & 6 & 0 & $\mathrm{Nd}$ & + & + & & & & & & \\
\hline Muscas et al. [27] & 2021 & 14 & 0 & $57.1 \%$ & + & + & & + & & & & + \\
\hline Muto et al. [93] & 2021 & 5 & 0 & 0 & $=$ & $=$ & & & & & & \\
\hline Rösler et al. [26] & 2021 & 39 & 0 & $69.2 \%$ & + & & & + & & - & & \\
\hline Rotermund et al. [95] & 2021 & 296 & 0 & 0 & + & + & + & + & & & $=$ & \\
\hline Shimizu et al. [96] & 2021 & 14 & 0 & 0 & & + & & + & & & & \\
\hline Yoon et al. [25] & 2021 & 11 & 9.1 & $18.2 \%$ & $=$ & + & & + & + & & - & \\
\hline
\end{tabular}




\section{Discussion}

There were over 1524 surgeries that reported using an exoscope: 1251 (72.7\%) on brain, whereas $274(15.9 \%)$ and $9(0.5 \%)$ on spine and peripheral nerves, respectively. Among these, more than 311 were gliomas, 171 were brain metastasis and 97 were meningioma and 244 were pituitary adenoma, 100 resulted ICH and 24 were neurovascular conflicts. Similarly in spine, 64 cervical and 189 lumbar pathologies were treated with the use of exoscope in the operative room, as well as 12 spine tumors were reported.

The development of surgical magnification and neurosurgery progressed on separate paths until the 1960s, when the merging of these two innovations led to the rapid growth of cerebral surgery [98-101]. From that time, intraoperative technological advances improved, and the $\mathrm{OM}$ and endoscope allowed complete resection of glioma and other intraventricular and pituitary tumors, neurovascular and spine diseases, under magnification with good lighting and through minimally invasive approaches [12,15,16,45,47,57,58,60,95,102,103].

Neuronavigation, ultrasound, intraoperative magnetic resonance imaging (MRI) and/ or computed tomography (CT) scan, robotic technology, augmented reality and awake surgery increased the ability of the neurosurgeon to perform safe and maximal tumor resection [12,102,104-110]. Exoscopes launched a new era in the field of neurosurgery. These exoscopes are designed to provide high-resolution 3D imaging of the structure of tissue, blood vessels and other features to enable more accurate surgery and, including a display video, allow for simultaneous surgical team viewing. Exoscopes represent the next generation of operative imaging, helping the neurosurgeon to operate in a more ergonomic sitting position, facilitating the surgery team and reducing surgeon fatigue by reducing the amount of time practitioners would have to view the images through a microscope eyepiece. These systems work to bridge the gap between OM and endoscopes by combining the form factor of the endoscope with the image quality of the microscope $[43,49,78,94]$. Some disadvantages of exoscopic visualization were reported, especially in the early 2D exoscope, such as a limited applicability in deep seated cranial pathologies and tissue identification in case of bleeding, a magnification of deep-seated pathologies and above all the lack of stereopsis. All of these disadvantages seem to be solved with new 3D exoscopes, which however led to headache and nausea in very few cases due to the use of polarized glasses $[19,41,51,52]$. Furthermore, these devices still have usage limitations due to their high cost and to the impossibility, at the moment, to use 5-ALA fluorescence for tumor resection. A major advantage of the exoscope is the shared $3 \mathrm{D}$ view for all participants in the procedure $[28,67,95]$. The possibility to look at the same time in the same monitor allows more than one surgeon to operate and improves efficiency by sharing information with all surgical staff. Although Takahashi and colleagues [61] reported that assistant surgeons could sometimes experience a rotated view of the monitor; in this case the use of two or more 3D monitors in the operative room can solve this problem.

One of the characteristics ascribed to exoscopes is that they are superior to a conventional $\mathrm{OM}$ in terms of ergonomic features both in brain and spine surgery $[37,39,49$, $52,67,69,90-92,94-97,111-115]$, as the ergonomic handling and the ease of intraoperative positioning of the device were found to be beneficial. Second, 3D monitors lead to an improved involvement of the co-surgeon and the scrub nurse during the procedure, and although some authors were satisfied with the high-resolution 3D digital images during surgery $[52,60,68,73]$, others were not satisfied with the visual quality. In spine surgery, when two neurosurgeons are operating facing each other, the use of 2 monitors each positioned in front of each surgeon allows extreme freedom of movement and modification of the surgical corridor [89]. The important aspect of the exoscope monitor is that the surgeon, assistant and nurse all see the same image with the same quality and the exoscope does not interfere with communication and allows all surgical staff to feel more involved in the surgical procedure [7]. By increasing the visualization of anatomic details helps to identify the different layers and the tumor-nerve interface, and exoscopes can be useful also for peripheral nerve sheath tumors to preserve functional fascicles achieving gross-total resection [88]. 
Exoscopic tools seem to shift from cortical cranial tumor surgery to deep-seated brain tumors, as exoscope technology has progressively improved during the last few years, with results in terms of clinical outcome and surgical complications similar to conventional OM [85]. Hafez and colleagues [23] reported the largest comparative and laboratory series with the use of the exoscope and OM and showed that both methods are effective in doing bypass suturing, whereas the suturing time was less using the microscope and stitch distribution was better using the exoscope. Among brain tumors, Gonen and colleagues [45] reported the largest series of glioma resection (56 patients) using the exoscope, accounting for 44 cases of high-grade gliomas and 12 of low-grade gliomas and reporting just one $(1.8 \%)$ perioperative complication (hemorrhage within the resection bed) in a patient with glioblastoma multiforme. Similarly, Gassie et al. [55], Piquer et al. [39], Day [44] and Eichberg et al. [30] reported that 30, 25, 22 and 12 patients, respectively, underwent surgical resection for glioma using different exoscopes. Overall postoperative surgical complications with permanent motor deficit range from $0 \%$ to $8 \%[30,39,44,55]$. Rotermund et al. [95] reported the largest series of patients underwent transsphenoidal surgery for pituitary adenoma (239 patients), reporting that no serious episodes or minor complications occurred based on the usage of the exoscope, as well as no significant differences regarding the duration of surgery, complications or extent of resection compared to conventional microscopy. Chen et al. [73] reported a total of 81 patients received tumor resection through the retrosigmoid approach with either an exoscope (39 patients) or an OM (42 patients). Patients in the two groups had comparable tumor location $(p=0.439)$ and Koos grading $(p=0.867)$. There were significant differences in the duration of surgery $(p=0.172)$, the extent of tumor resection ( $p=0.858)$, facial function $(p=0.838)$ and hearing ability $(p=1.000)$. Gonen et al. [45], Khalessi et al. [58], Ahmad et al. [9] had a total of 35 patients with neurovascular pathologies (aneurysms, arteriovenous malformations, cavernomas) who underwent surgery with an exoscope, reporting an overall good outcome and only $2.8 \%$ postoperative complications. In particular, Ahmad et al. [9] reported 12 microvascular anastomosis, reporting no difference in operative time $(p=0.714)$, ischemia time $(p=0.972)$, or microsurgical complications $(p=1)$ between the ORBEYE and conventional microscopy groups.

Regarding 3D visualization, Ricciardi et al. [14] in a previous review comparing exoscopes and microscopes found that image quality, optical power and magnification of the exoscope were rated at least equivalent to the microscope. In addition, exoscopes are also able to allow the surgeons to quickly switch from a micro to a macro vision and vice versa, when necessary, to explore all corners of the surgical field and to keep an eye on any bleeding $[35,39,52]$. Nevertheless, at present exoscopes have some limitations. Burkhardt et al. [71] reported that in 5 out of 10 cases (50\%) of cranial surgery, a switch to the OM was necessary, due to the need to use 5-ALA fluorescence guided visualization in two cases and because the illumination of the depth of the operative field was not sufficient in 3 cases. Lin et al. [81] obtained gross total resection in all four cases of intraventricular meningiomas, reporting no intraoperative complications nor conversion to microscopic or open approach. Ridge et al. [116] and Teo et al. [29] highlighted the role of the exoscope in reducing the risk of infection exposure to the surgical team during the COVID-19 pandemic [117-119].

The use of the exoscope has been largely reported with a variety of different exoscope models used also in spinal surgery $[5,7,15,26,29,41,71,89]$. Ariffin et al. [15] submitted an interesting series of minor to major surgical spine procedures in 69 patients using the exoscope, reporting only four cases (5.8\%) of dural tear as surgical complications and no postoperative neurological deficits. Similarly, Siller et al. [5] (40 patients undergoing lumbar posterior decompression (LPD) and 20 patients undergoing anterior cervical discectomy and fusion (ACDF), showing no intraoperative complications by using the exoscope, and reported similar results in outcome compared to controls in whom an OM was used. According to the attending surgeon, the intraoperative handling of the instruments was rated to be comparable to that of the OM, while the comfort level of the surgeon's posture intraoperatively (especially during "undercutting" procedures) was rated as superior [15]. 
Otherwise, Burkhardt et al. [71], including 16 cranial and 18 spinal surgical procedures in their paper, reported some intraoperative difficulties and that one spinal and five cranial procedures switched to OM or the endoscope for the following reasons: poor illumination (four cases), tissue identification (one case), and the need for fluorescence imaging (one case).

This review shows how a such large number of published papers and patients underwent brain and spinal surgery with the exoscope, showing the simplicity of use, the total safety for the patient, the good 3D vision and magnification of the surgical field and the opportunity of better interaction with other members of the surgical staff. All these points set the first step for subsequent and short-term changes in the field of neurosurgery and offer new educational possibilities for young neurosurgery and medical students. This review has some limitations. First, this review is susceptible to changes over the short term, as exoscopes were increasingly used in recent years and therefore an increasing number of papers will be published in the near future, and because technology and science advance incessantly. Second, this review aims to show the advantages and disadvantages of a new tool used in neurosurgery, reporting surgical experiences of different authors and summarizing the current literature, without drawing unique technical conclusions, as we believe it is still too early at this moment. Future clinical studies and reviews are needed to demonstrate if exoscopes will change the neurosurgical sciences.

\section{Conclusions}

Exoscopes have been used constantly in an increasing number of surgical procedures all around the world, suggesting that they could ultimately replace the OM in the future and represent the beginning of a new era of intraoperative visualization in neurosurgery. A 3D exoscope seems to be a safe alternative compared to the OM for most common brain and spinal procedures, with several advantages that have been reached. This review confirmed the role of the exoscope as a new tool that can help surgeons during surgery and even replace the $\mathrm{OM}$ in the near future due to several aspects: a better ergonomic posture of the surgeons during surgical procedures, the possibility to improve neurosurgical education, and in creating a better and effective operational team involvement. The quality of images and $3 \mathrm{D} 4 \mathrm{~K}$ videos in most recent exoscopes has been increasingly improved in recent years, although at the moment the most reported drawback remains the slight lack of depth perception. The exoscope itself can be considered a useful educational tool in neurosurgery. As with other adaptations of new technology, it will take some time for systems to be tweaked and the pros and cons of different approaches to be better appreciated. More research needs to be done. A short learning curve is mandatory.

Author Contributions: Conceptualization, N.M. and L.R.; methodology, N.M. and A.S.; validation, N.M. and A.S.; data curation, N.M.; writing—original draft preparation, N.M.; writing-review and editing, N.M., L.R. and G.T.; supervision, N.M. and G.T. All authors have read and agreed to the published version of the manuscript.

Funding: This research received no external funding.

Institutional Review Board Statement: Not applicable.

Informed Consent Statement: Not applicable.

Acknowledgments: We thank Federica Tataranni for her English revision.

Conflicts of Interest: The authors declare no conflict of interest.

\section{References}

1. Kriss, T.C.; Kriss, V.M. History of the operating microscope: From magnifying glass to microneurosurgery. Neurosurgery 1998, 42 , 899-907. [CrossRef] [PubMed]

2. $\quad$ Langer, D.J.; White, T.G.; Schulder, M.; Boockvar, J.A.; Labib, M.; Lawton, M.T. Advances in intraoperative optics: A brief review of current exoscope platforms. Oper. Neurosurg. 2020, 19, 84-93. [CrossRef] [PubMed] 
3. Fuse, Y.; Watanabe, T.; Shintai, K.; Kishida, Y.; Nagatani, T.; Seki, Y. Non-brain-exposure exoscopic and endoscopic volume reduction surgery for benign meningioma en plaque in an elderly patient: A case report. NMC Case Rep. J. 2020, 7, 233-236. [CrossRef] [PubMed]

4. Angileri, F.F.; Esposito, F.; Scibilia, A.; Priola, S.M.; Raffa, G.; German, A. Exoscope-guided (VITOM 3D) single-stage removal of supratentorial cavernous angioma and hemangioblastoma: 3-dimensional operative video. Oper. Neurosurg. 2019, 17, E164-E165. [CrossRef]

5. Siller, S.; Zoellner, C.; Fuetsch, M.; Trabold, R.; Tonn, J.C.; Zausinger, S. A high-definition 3D exoscope as an alternative to the operating microscope in spinal microsurgery. J. Neurosurg. Spine 2020, 33, 705-714. [CrossRef]

6. De Divitiis, O.; D'avella, E.; Denaro, L.; Somma, T.; Sacco, M.; D'avella, D. Vitom 3D: Preliminary experience with intradural extramedullary spinal tumors. J. Neurosurg. Sci. 2019, 2, 1-4. [CrossRef]

7. Muhammad, S.; Lehecka, M.; Niemelä, M. Preliminary experience with a digital robotic exoscope in cranial and spinal surgery: A review of the Synaptive Modus V system. Acta Neurochir. 2019, 161, 2175-2180. [CrossRef]

8. Sindelar, B.D.; Patel, V.; Chowdhry, S.; Bailes, J.E. A case report in hemorrhagic stroke: A complex disease process and requirement for a multimodal treatment approach. Cureus 2018, 10, e2976. [CrossRef]

9. Ahmad, F.I.; Mericli, A.F.; DeFazio, M.V.; Chang, E.I.; Hanasono, M.M.; Pederson, W.C.; Kaufman, M.; Selber, J.C. Application of the ORBEYE three-dimensional exoscope for microsurgical procedures. Microsurgery 2020, 40, 468-472. [CrossRef]

10. Baron, R.B.; Lakomkin, N.; Schupper, A.J.; Nistal, D.; Nael, K.; Price, G.; Hadjipanayis, C.G. Postoperative outcomes following glioblastoma resection using a robot-assisted digital surgical exoscope: A case series. J. Neurooncol. 2020, 148, 519-527. [CrossRef]

11. Barkun, J.S.; Aronson, J.K.; Feldman, L.S.; Maddern, G.J.; Strasberg, S.M.; Altman, D.G.; Barkun, J.S.; Blazeby, J.M.; Boutron, I.C.; Campbell, W.B.; et al. Evaluation and stages of surgical innovations. Lancet 2009, 374, 1089-1096. [CrossRef]

12. Raheja, A.; Mishra, S.; Garg, K.; Katiyar, V.; Sharma, R.; Tandon, V.; Goda, R.; Suri, A.; Kale, S.S. Impact of different visualization devices on accuracy, efficiency, and dexterity in neurosurgery: A laboratory investigation. Neurosurg. Focus 2021, 50, E18. [CrossRef]

13. Sack, J.; Steinberg, J.A.; Rennert, R.C.; Hatefi, D.; Pannell, J.S.; Levy, M.; Khalessi, A.A. Initial experience using a high-definition 3-dimensional exoscope system for microneurosurgery. Oper. Neurosurg. 2018, 14, 395-401. [CrossRef]

14. Ricciardi, L.; Chaichana, K.L.; Cardia, A.; Stifano, V.; Rossini, Z.; Olivi, A.; Sturiale, C.L. The exoscope in neurosurgery: An innovative "point of view". A systematic review of the technical, surgical and educational aspects. World Neurosurg. 2019, 124, 136-144. [CrossRef]

15. Ariffin, M.H.M.; Ibrahim, K.; Baharudin, A.; Tamil, A.M. Early Experience, setup, learning curve, benefits, and complications associated with exoscope and three-dimensional $4 \mathrm{~K}$ hybrid digital visualizations in minimally invasive spine surgery. Asian Spine J. 2020, 14, 59-65. [CrossRef]

16. Garneau, J.C.; Laitman, B.M.; Cosetti, M.K.; Hadjipanayis, C.; Wanna, G. The use of the exoscope in lateral skull base surgery: Advantages and limitations. Otol. Neurotol. 2019, 40, 236-240. [CrossRef]

17. Nishiyama, K. From Exoscope into the next generation. J. Korean Neurosurg. Soc. 2017, 60, 289-293. [CrossRef]

18. Corsalini, M.; Di Venere, D.; Sportelli, P.; Magazzino, D.; Ripa, C.; Cantatore, F.; Cagnetta, G.; De Rinaldis, C.; Montemurro, N.; De Giacomo, A.; et al. Evaluation of prosthetic quality and masticatory efficiency in patients with total removable prosthesis: Study of 12 cases. Oral Implantol. 2018, 11, 230-240.

19. Shirzadi, A.; Mukherjee, D.; Drazin, D.G.; Paff, M.; Perri, B.; Mamelak, A.N.; Siddique, K. Use of the video telescope operating monitor (VITOM) as an alternative to the operating microscope in spine surgery. Spine 2012, 37, E1517-E1523. [CrossRef]

20. Ricciardi, L.; Mattogno, P.P.; Olivi, A.; Sturiale, C.L. Exoscope era: Next technical and educational step in microneurosurgery. World Neurosurg. 2019, 128, 371-373. [CrossRef]

21. Wali, A.R.; Kang, K.M.; Rennert, R.; Santiago-Dieppa, D.; Khalessi, A.A.; Levy, M. First-in-human clinical experience using high-definition exoscope with intraoperative indocyanine green for clip reconstruction of unruptured large pediatric aneurysm. World Neurosurg. 2021, 151, 52. [CrossRef]

22. Dawley, T.; Schulder, M. Commentary: First-in-man clinical experience using a high-definition 3-dimensional exoscope system for microneurosurgery. Oper. Neurosurg. 2019, 16, E161-E162. [CrossRef]

23. Hafez, A.; Elsharkawy, A.; Schwartz, C.; Muhammad, S.; Laakso, A.; Niemelä, M.; Lehecka, M. Comparison of conventional microscopic and exoscopic experimental bypass anastomosis: A technical analysis. World Neurosurg. 2020, 135, e293-e299. [CrossRef]

24. Perrini, P.; Montemurro, N.; Caniglia, M.; Lazzarotti, G.; Benedetto, N. Wrapping of intracranial aneurysms: Single-center series and systematic review of the literature. Br. J. Neurosurg. 2015, 29, 785-791. [CrossRef]

25. Yoon, W.S.; Lho, H.W.; Chung, D.S. Evaluation of 3-dimensional exoscopes in brain tumor surgery. J. Korean Neurosurg. Soc. 2021, 64, 289-296. [CrossRef]

26. Rösler, J.; Georgiev, S.; Roethe, A.L.; Chakkalakal, D.; Acker, G.; Dengler, N.F.; Prinz, V.; Hecht, N.; Faust, K.; Schneider, U.; et al. Clinical implementation of a 3D4K-exoscope (Orbeye) in microneurosurgery. Neurosurg. Rev. 2021, 18, 1-9. [CrossRef]

27. Muscas, G.; Battista, F.; Boschi, A.; Morone, F.; Della Puppa, A. A Single-center experience with the olympus ORBEYE 4K-3D exoscope for microsurgery of complex cranial cases: Technical nuances and learning curve. J. Neurol. Surg. Part A Cent. Eur. Neurosurg. 2021, 82, 484-489. [CrossRef] 
28. Hafez, A.; Haeren, R.H.L.; Dillmann, J.; Laakso, A.; Niemelä, M.; Lehecka, M. Comparison of operating microscope and exoscope in a highly challenging experimental setting. World Neurosurg. 2021, 147, e468-e475. [CrossRef] [PubMed]

29. Teo, T.H.L.; Tan, B.J.; Loo, W.L.; Yeo, A.K.S.; Dinesh, S.K. Utility of a high-definition 3D digital exoscope for spinal surgery during the COVID-19 pandemic. Bone Jt. Open 2020, 1, 359-363. [CrossRef] [PubMed]

30. Eichberg, D.G.; Di, L.; Shah, A.H.; Luther, E.M.; Jackson, C.; Marenco-Hillembrand, L.; Chaichana, K.L.; Ivan, M.E.; Starke, R.M.; Komotar, R.J. Minimally invasive resection of intracranial lesions using tubular retractors: A large, multi-surgeon, multiinstitutional series. J. Neurooncol. 2020, 149, 35-44. [CrossRef] [PubMed]

31. Moher, D.; Shamseer, L.; Clarke, M.; Ghersi, D.; Liberati, A.; Petticrew, M.; Shekelle, P.; Stewart, L.A.; PRISMA-P Group. Preferred reporting items for systematic review and meta-analysis protocols (PRISMA-P) 2015 statement. Syst. Rev. 2015, 4, 1. [CrossRef]

32. Gildenberg, P.L.; Labuz, J. Stereotactic craniotomy with the exoscope. Stereotact. Funct. Neurosurg. 1997, 68, 64-71. [CrossRef]

33. Mamelak, A.N.; Danielpour, M.; Black, K.L.; Hagike, M.; Berci, G. A high-definition exoscope system for neurosurgery and other microsurgical disciplines: Preliminary report. Surg. Innov. 2008, 15, 38-46. [CrossRef]

34. Mamelak, A.N.; Nobuto, T.; Berci, G. Initial clinical experience with a high-definition exoscope system for microneurosurgery. Neurosurgery 2010, 67, 476-483. [CrossRef]

35. Di leva, A.; Komatsu, M.; Komatsu, F.; Tschabitscher, M. Endoscopic telovelar approach to the fourth ventricle: Anatomic study. Neurosurg. Rev. 2012, 35, 341-348. [CrossRef]

36. Mamelak, A.N.; Drazin, D.; Shirzadi, A.; Black, K.L.; Berci, G. Infratentorial supracerebellar resection of a pineal tumor using a high definition video exoscope $\left(\right.$ VITOM $\left.^{\circledR}\right)$. J. Clin. Neurosci. 2012, 19, 306-309. [CrossRef]

37. Belloch, J.P.; Rovira, V.; Llácer, J.L.; Riesgo, P.A.; Cremades, A. Fluorescence-guided surgery in high grade gliomas using an exoscope system. Acta Neurochir. 2014, 156, 653-660. [CrossRef]

38. Birch, K.; Drazin, D.; Black, K.L.; Williams, J.; Berci, G.; Mamelak, A.N. Clinical experience with a high definition exoscope system for surgery of pineal region lesions. J. Clin. Neurosci. 2014, 21, 1245-1249. [CrossRef]

39. Piquer, J.; Llácer, J.L.; Rovira, V.; Riesgo, P.; Rodriguez, R.; Cremades, A. Fluorescence-guided surgery and biopsy in gliomas with an exoscope system. Biomed Res. Int. 2014, 2014, 207974. [CrossRef]

40. Ritsma, B.; Kassam, A.; Dowlatshahi, D.; Nguyen, T.; Stotts, G. Minimally Invasive Subcortical Parafascicular Transsulcal Access for Clot Evacuation (Mi SPACE) for Intracerebral Hemorrhage. Case Rep. Neurol. Med. 2014, 2014, 102307. [CrossRef]

41. Parihar, V.; Yadav, Y.R.; Kher, Y.; Ratre, S.; Sethi, A.; Sharma, D. Learning neuroendoscopy with an exoscope system (video telescopic operating monitor): Early clinical results. Asian J. Neurosurg. 2016, 11, 421-426. [CrossRef]

42. Scranton, R.A.; Fung, S.H.; Britz, G.W. Transulcal parafascicular minimally invasive approach to deep and subcortical cavernomas: Technical note. J. Neurosurg. 2016, 125, 1360-1366. [CrossRef]

43. Bauer, A.M.; Rasmussen, P.A.; Bain, M.D. Initial single-center technical experience with the brainpath system for acute intracerebral hemorrhage evacuation. Oper. Neurosurg. 2017, 13, 69-76. [CrossRef]

44. Day, J.D. Transsulcal parafascicular surgery using brain path ${ }^{\circledR}$ for subcortical lesions. Neurosurgery 2017, 64, 151-156. [CrossRef]

45. Gonen, L.; Chakravarthi, S.S.; Monroy-Sosa, A.; Celix, J.M.; Kojis, N.; Singh, M.; Jennings, J.; Fukui, M.B.; Rovin, R.A.; Kassam, A.B. Initial experience with a robotically operated video optical telescopic-microscope in cranial neurosurgery: Feasibility, safety, and clinical applications. Neurosurg. Focus 2017, 42, E9. [CrossRef]

46. Jackson, C.; Gallia, G.L.; Chaichana, K.L. Minimally invasive biopsies of deep-seated brain lesions using tubular retractors under exoscopic visualization. J. Neurol. Surg. A Cent. Eur. Neurosurg. 2017, 78, 588-594. [CrossRef]

47. Krishnan, K.G.; Schöller, K.; Uhl, E. Application of a compact high-definition exoscope for illumination and magnification in high-precision surgical procedures. World Neurosurg. 2017, 97, 652-660. [CrossRef]

48. Labib, M.A.; Shah, M.; Kassam, A.B.; Young, R.; Zucker, L.; Maioriello, A.; Britz, G.; Agbi, C.; Day, J.D.; Gallia, G.; et al. The safety and feasibility of image-guided brainpath-mediated transsulcul hematoma evacuation: A multicenter study. Neurosurgery 2017, 80, 515-524. [CrossRef]

49. Moisi, M.D.; Hoang, K.; Tubbs, R.S.; Page, J.; Fisahn, C.; Paulson, D.; Jeyamohan, S.; Delashaw, J.; Hanscom, D.; Oskouian, R.J.; et al. Advancement of surgical visualization methods: Comparison study between traditional microscopic surgery and a novel robotic optoelectronic visualization tool for spinal surgery. World Neurosurg. 2017, 98, 273-277. [CrossRef]

50. Nagata, Y.; Watanabe, T.; Nagatani, T.; Takeuchi, K.; Chu, J.; Wakabayashi, T. The multiscope technique for microvascular decompression. World Neurosurg. 2017, 103, 310-314. [CrossRef]

51. Oertel, J.M.; Burkhardt, B.W. Vitom-3D for exoscopic neurosurgery: Initial experience in cranial and spinal procedures. World Neurosurg. 2017, 105, 153-162. [CrossRef] [PubMed]

52. Rossini, Z.; Cardia, A.; Milani, D.; Lasio, G.B.; Fornari, M.; D’Angelo, V. VITOM 3D: Preliminary experience in cranial surgery. World Neurosurg. 2017, 107, 663-668. [CrossRef] [PubMed]

53. Weiner, H.L.; Placantonakis, D.G. Resection of a pediatric thalamic juvenile pilocytic astrocytoma with whole brain tractography. Cureus 2017, 9, e1768. [CrossRef] [PubMed]

54. Beez, T.; Munoz-Bendix, C.; Beseoglu, K.; Steiger, H.J.; Ahmadi, S.A. First clinical applications of a high-definition threedimensional exoscope in pediatric neurosurgery. Cureus 2018, 10, e2108. [CrossRef]

55. Gassie, K.; Wijesekera, O.; Chaichana, K.L. Minimally invasive tubular retractor-assisted biopsy and resection of subcortical intra-axial gliomas and other neoplasms. J. Neurosurg. Sci. 2018, 62, 682-689. [CrossRef] 
56. Griessenauer, C.; Medin, C.; Goren, O.; Schirmer, C.M. Image-guided, minimally invasive evacuation of intracerebral hematoma: A matched cohort study comparing the endoscopic and tubular exoscopic systems. Cureus 2018, 10, e3569. [CrossRef]

57. Iyer, R.; Chaichana, K.L. Minimally invasive resection of deep-seated high-grade gliomas using tubular retractors and exoscopic visualization. J. Neurol. Surg. A Cent. Eur. Neurosurg. 2018, 79, 330-336. [CrossRef]

58. Khalessi, A.A.; Rahme, R.; Rennert, R.C.; Borgas, P.; Steinberg, J.A.; White, T.G.; Santiago-Dieppa, D.R.; Boockvar, J.A.; Hatefi, D.; Pannell, J.S.; et al. First-in-man clinical experience using a high-definition 3-dimensional exoscope system for microneurosurgery. Oper. Neurosurg. 2019, 16, 717-725. [CrossRef]

59. Klinger, D.R.; Reinard, K.A.; Ajayi, O.O.; Delashaw, J.B., Jr. Microsurgical clipping of an anterior communicating artery aneurysm using a novel robotic visualization tool in lieu of the binocular operating microscope: Operative video. Oper. Neurosurg. 2018, 14, 26-28. [CrossRef]

60. Mampre, D.; Bechtle, A.; Chaichana, K.L. Minimally invasive resection of intra-axial posterior fossa tumors using tubular retractors. World Neurosurg. 2018, 119, e1016-e1020. [CrossRef]

61. Takahashi, S.; Toda, M.; Nishimoto, M.; Ishihara, E.; Miwa, T.; Akiyama, T.; Horiguchi, T.; Sasaki, H.; Yoshida, K. Pros and cons of using ORBEYE ${ }^{\mathrm{TM}}$ for microneurosurgery. Clin. Neurol. Neurosurg. 2018, 174, 57-62. [CrossRef]

62. Akbari, S.H.A.; Sylvester, P.T.; Kulwin, C.; Shah, M.V.; Somasundaram, A.; Kamath, A.A.; Beaumont, T.L.; Rich, K.M.; Chicoine, M.R. Initial experience using intraoperative magnetic resonance imaging during a trans-sulcal tubular retractor approach for the resection of deep-seated brain tumors: A case series. Oper. Neurosurg. 2019, 16, 292-301. [CrossRef]

63. Bakhsheshian, J.; Strickland, B.A.; Jackson, C.; Chaichana, K.L.; Young, R.; Pradilla, G.; Chen, J.W.; Bailes, J.; Zada, G. Multicenter investigation of channel-based subcortical trans-sulcal exoscopic resection of metastatic brain tumors: A retrospective case series. Oper. Neurosurg. 2019, 16, 159-166. [CrossRef]

64. Herlan, S.; Marquardt, J.S.; Hirt, B.; Tatagiba, M.; Ebner, F.H. 3D exoscope system in neurosurgery-comparison of a standard operating microscope with a new 3d exoscope in the cadaver lab. Oper. Neurosurg. 2019, 17, 518-524. [CrossRef]

65. Kwan, K.; Schneider, J.R.; Du, V.; Falting, L.; Boockvar, J.A.; Oren, J.; Levine, M.; Langer, D.J. Lessons learned using a highdefinition 3-dimensional exoscope for spinal surgery. Oper. Neurosurg. 2019, 16, 619-625. [CrossRef]

66. Li Ching Ng, A.; Di Ieva, A. How I do it: 3D exoscopic endoscope-assisted microvascular decompression. Acta Neurochir. 2019, 161, 1443-1447. [CrossRef]

67. Murai, Y.; Sato, S.; Yui, K.; Morimoto, D.; Ozeki, T.; Yamaguchi, M.; Tateyama, K.; Nozaki, T.; Tahara, S.; Yamaguchi, F.; et al. Preliminary clinical microneurosurgical experience with the 4K3-dimensional microvideoscope (ORBEYE) system for microneurological surgery: Observation study. Oper. Neurosurg. 2019, 16, 707-716. [CrossRef]

68. Nossek, E.; Schneider, J.R.; Kwan, K.; Kulason, K.O.; Du, V.; Chakraborty, S.; Rahme, R.; Faltings, L.; Ellis, J.; Ortiz, R.; et al. Technical aspects and operative nuances using a high-definition 3-dimensional exoscope for cerebral bypass surgery. Oper. Neurosurg. 2019, 17, 157-163. [CrossRef]

69. Smith, S.; Kozin, E.D.; Kanumuri, V.V.; Barber, S.R.; Backous, D.; Flávio Nogueira, J.; Lee, D.J. Initial experience with 3-dimensional exoscope-assisted transmastoid and lateral skull base surgery. Otolaryngol. Head Neck. Surg. 2019, 160, 364-367. [CrossRef]

70. Barbagallo, G.M.V.; Certo, F. Three-dimensional, high-definition exoscopic anterior cervical discectomy and fusion: A valid alternative to microscope-assisted surgery. World Neurosurg. 2019, 130, e244-e250. [CrossRef]

71. Burkhardt, B.W.; Csokonay, A.; Oertel, J.M. 3D-exoscopic visualization using the VITOM-3D in cranial and spinal neurosurgery. What are the limitations? Clin. Neurol. Neurosurg. 2020, 198, 106101. [CrossRef]

72. Chakravarthi, S.S.; Lyons, L.; Bercu, M.; Singer, J.A. Minimally invasive parafascicular surgical approach for the management of a pediatric third ventricular ependymoma: Case report and review of literature. World Neurosurg. 2020, 141, 311-317. [CrossRef]

73. Chen, X.; Gao, X.L.; Chai, Y.; Shi, M.M.; Zhang, J.N.; Yue, S.Y. Use of a compact high-definition two-dimensional exoscope in surgical treatment of large vestibular schwannoma. Chin. Med. J. 2020, 133, 1292-1297. [CrossRef]

74. Crosetti, E.; Arrigoni, G.; Manca, A.; Fantini, M.; Caracciolo, A.; Sardanapoli, F.; Succo, G. VITOM-3D assisted neck dissection via a retroauricular approach (RAND-3D): A preclinical investigation in a cadaver lab. Acta Otorhinolaryngol. Ital. 2020, 40, 343-351. [CrossRef]

75. D'Ercole, M.; Serchi, E.; Zanello, M.; Tufo, T.; Sturiale, C. Clinical application of a high definition three-dimensional exoscope in anterior lumbar interbody fusion: Technical note. Int. J. Spine Surg. 2020, 14, 1003-1008. [CrossRef]

76. Doglietto, F.; Belotti, F.; Panciani, P.; Poliani, P.L.; Fontanella, M.M. High-definition 3-dimensional exoscope for 5-ALA glioma surgery: 3-dimensional operative video. Oper. Neurosurg. 2020, 18, E82. [CrossRef]

77. Garneau, J.C.; Laitman, B.M.; Cosetti, M.K.; Hadjipanayis, C.; Wanna, G.B. Repair of a temporal bone encephalocele with the surgical exoscope. Otol. Neurotol. 2020, 41, 561. [CrossRef] [PubMed]

78. Khatri, D.; Wagner, K.; Ligas, B.; Higbie, C.; Langer, D. Excision of a retrochiasmatic craniopharyngioma by transcallosal, interforniceal approach with exoscope assistance: 2-dimensional operative video. Oper. Neurosurg. 2020, 19, E411. [CrossRef]

79. Kleshchova, O.; White, T.G.; Kwan, K.; Chiluwal, A.; Anderson, T.A.; Langer, D.J. Resection of a posterior fossa endodermal cyst with exoscopic assistance: 2-dimensional operative video. Oper. Neurosurg. 2020, 18, E173-E174. [CrossRef]

80. Ligas, B.; Khatri, D.; Higbie, C.; Wagner, K.; Langer, D. Hemifacial spasm due to bony stenosis of the internal auditory meatus: Look beyond the loop. World Neurosurg. 2020, 137, 179-182. [CrossRef] 
81. Lin, M.; Bakhsheshian, J.; Strickland, B.; Rennert, R.C.; Chu, R.M.; Chaichana, K.L.; Zada, G. Exoscopic resection of atrial intraventricular meningiomas using a navigation-assisted channel-based trans-sulcal approach: Case series and literature review. J. Clin. Neurosci. 2020, 71, 58-65. [CrossRef] [PubMed]

82. Oren, J.; Kwan, K.; Schneider, J.; Levine, M.; Langer, D. Minimally invasive navigated foraminal discectomy via contralateral approach using a 3-dimensional 4k high-definition exoscope: 2-dimensional operative video. Oper. Neurosurg. 2020, 19 , E188. [CrossRef] [PubMed]

83. Pafitanis, G.; Hadjiandreou, M.; Alamri, A.; Uff, C.; Walsh, D.; Myers, S. The Exoscope versus operating microscope in microvascular surgery: A simulation non-inferiority trial. Arch. Plast. Surg. 2020, 47, 242-249. [CrossRef] [PubMed]

84. Patel, N.V.; Ligas, B.; Gandhi, S.; Ellis, J.; Ortiz, R.; Costantino, P.; Qato, K.; Langer, D.J. Internal maxillary to middle cerebral artery bypass using an anterior tibial artery graft, performed using a 3-dimensional exoscope: 2-dimensional operative video. Oper. Neurosurg. 2020, 19, E187. [CrossRef]

85. Roethe, A.L.; Landgraf, P.; Schröder, T.; Misch, M.; Vajkoczy, P.; Picht, T. Monitor-based exoscopic 3D4k neurosurgical interventions: A two-phase prospective-randomized clinical evaluation of a novel hybrid device. Acta Neurochir. 2020, 162, $2949-2961$. [CrossRef]

86. Rubini, A.; Di Gioia, S.; Marchioni, D. 3D exoscopic surgery of lateral skull base. Eur. Arch. Otorhinolaryngol. 2020, 277, 687-694. [CrossRef]

87. Silverstein, J.W.; Ellis, J.A.; Langer, D.J. Loss of motor evoked potentials due to carotid artery retraction in an exoscopic clipping of a basilar tip aneurysm. Neurodiagn. J. 2020, 60, 289-299. [CrossRef]

88. Vetrano, I.G.; Acerbi, F.; Falco, J.; D’Ammando, A.; Devigili, G.; Nazzi, V. High-definition 4K 3D exoscope (ORBEYETM) in peripheral nerve sheath tumor surgery: A preliminary, explorative, pilot study. Oper. Neurosurg. 2020, 19, 480-488. [CrossRef]

89. Visocchi, M.; Mattogno, P.P.; Ciappetta, P.; Barbagallo, G.; Signorelli, F. Combined transoral exoscope and OArm-assisted approach for craniovertebral junction surgery: Light and shadows in single-center experience with improving technologies. J. Craniovertebr. Junction Spine 2020, 11, 293-299.

90. Amoo, M.; Henry, J.; Javadpour, M. Beyond magnification and illumination: Preliminary clinical experience with the 4K 3D ORBEYETM exoscope and a literature review. Acta Neurochir. 2021, 163, 2107-2115. [CrossRef]

91. Kim, M.; Wainwright, J.; Stein, A.; Hanft, S. Posterior transdural approach for a calcified thoracic intradural disc herniation using a 3-dimensional exoscope: 2-dimensional operative video. Oper. Neurosurg. 2021, 21, E44-E45. [CrossRef]

92. Marenco-Hillembrand, L.; Suarez-Meade, P.; Chaichana, K.L. Bur hole-based resections of intrinsic brain tumors with exoscopic visualization. J. Neurol. Surg. A Cent. Eur. Neurosurg. 2021, 82, 105-111. [CrossRef]

93. Muto, J.; Mine, Y.; Nakagawa, Y.; Joko, M.; Kagami, H.; Inaba, M.; Hasegawa, M.; Lee, J.Y.K.; Hirose, Y. Intraoperative real-time near-infrared optical imaging for the identification of metastatic brain tumors via microscope and exoscope. Neurosurg. Focus 2021, 50, E11. [CrossRef]

94. Rennert, R.C.; Khani, M.; Thomas, K.; Morris, T.W.; Rodriguez, A.; Day, J.D. Transsulcal parafascicular brain path-assisted approach to subcortical lesions: 2-dimensional operative video. Surg. Neurol. Int. 2021, 17, 107.

95. Rotermund, R.; Regelsberger, J.; Osterhage, K.; Aberle, J.; Flitsch, J. 4K 3-dimensional video microscope system (orbeye) for transsphenoidal pituitary surgery. Acta Neurochir. 2021, 163, 2097-2106. [CrossRef]

96. Shimizu, T.; Toyota, S.; Nakagawa, K.; Murakami, T.; Mori, K.; Kishima, H.; Taki, T. Retrosigmoid approach in the supine position using ORBEYE: A consecutive series of 14 cases. Neurol. Med. Chir. 2021, 61, 55-61. [CrossRef]

97. Strickland, B.A.; Brunswick, A.; Zada, G. Exoscopic to endoscopic channel-based trans-sulcal resection of a third ventricular cavernous malformation: Technical case illustration. World Neurosurg. 2021, 148, 66. [CrossRef]

98. Yaşargil, M.G.; Krayenbühl, H. The use of the binocular microscope in neurosurgery. Bibl. Ophthalmol. 1970, 81, $62-65$.

99. Uluç, K.; Kujoth, G.C.; Başkaya, M.K. Operating microscopes: Past, present, and future. Neurosurg. Focus 2009, 27, E4. [CrossRef]

100. Perrini, P.; Montemurro, N.; Iannelli, A. The contribution of Carlo Giacomini (1840-1898): The limbus Giacomini and beyond. Neurosurgery 2013, 72, 475-482. [CrossRef]

101. Hayden, M.G.; Lee, M.; Guzman, R.; Steinberg, G.K. The evolution of cerebral revascularization surgery. Neurosurg. Focus 2009, 26, E17. [CrossRef]

102. Zebian, B.; Vergani, F.; Lavrador, J.P.; Mukherjee, S.; Kitchen, W.J.; Stagno, V.; Chamilos, C.; Pettorini, B.; Mallucci, C. Recent technological advances in pediatric brain tumor surgery. CNS Oncol. 2017, 6, 71-82. [CrossRef]

103. Montemurro, N.; Fanelli, G.N.; Scatena, C.; Ortenzi, V.; Pasqualetti, F.; Mazzanti, C.M.; Morganti, R.; Paiar, F.; Naccarato, A.G.; Perrini, P. Surgical outcome and molecular pattern characterization of recurrent glioblastoma multiforme: A single-center retrospective series. Clin. Neurol. Neurosurg. 2021, 207, 106735. [CrossRef]

104. Rahman, M.; Murad, G.J.; Bova, F.; Friedman, W.A.; Mocco, J. Stereotactic radiosurgery and the linear accelerator: Accelerating electrons in neurosurgery. Neurosurg. Focus 2009, 27, E13. [CrossRef]

105. Montemurro, N.; Condino, S.; Cattari, N.; D'Amato, R.; Ferrari, V.; Cutolo, F. Augmented reality-assisted craniotomy for parasagittal and convexity en plaque meningiomas and custom-made cranio-plasty: A preliminary laboratory report. Int. J. Environ. Res. Public Health 2021, 18, 9955. [CrossRef]

106. Shurkhay, V.A.; Goryaynov, S.A.; Aleksandrova, E.V.; Spallone, A.; Potapov, A.A. Navigation systems in neurosurgery. Vopr Neirokhirurgii Im NN Burdenko 2016, 80, 107-114. [CrossRef] 
107. Condino, S.; Montemurro, N.; Cattari, N.; D'Amato, R.; Thomale, U.; Ferrari, V.; Cutolo, F. Evaluation of a wearable AR platform for guiding complex craniotomies in neurosurgery. Ann. Biomed Eng. 2021, 49, 2590-2605. [CrossRef]

108. Trevisi, G.; Roujeau, T.; Duffau, H. Awake surgery for hemispheric low-grade gliomas: Oncological, functional and methodological differences between pediatric and adult populations. Child's Nerv. Syst. 2016, 32, 1861-1874. [CrossRef]

109. Montemurro, N.; Herbet, G.; Duffau, H. Right cortical and axonal structures eliciting ocular deviation during electrical stimulation mapping in awake patients. Brain Topogr. 2016, 29, 561-571. [CrossRef]

110. Perrini, P.; Gambacciani, C.; Martini, C.; Montemurro, N.; Lepori, P. Anterior cervical corpectomy for cervical spondylotic myelopathy: Reconstruction with expandable cylindrical cage versus iliac crest autograft. A retrospective study. Clin. Neurol. Neurosurg. 2015, 139, 258-263. [CrossRef]

111. Lavé, A.; Gondar, R.; Demetriades, A.K.; Meling, T.R. Ergonomics and musculoskeletal disorders in neurosurgery: A systematic review. Acta Neurochir. 2020, 162, 2213-2220. [CrossRef] [PubMed]

112. Canseco, J.A.; Schroeder, G.D.; Patel, P.D.; Grasso, G.; Chang, M.; Kandziora, F.; Vialle, E.N.; Oner, F.C.; Schnake, K.J.; Dvorak, M.F.; et al. Regional and experiential differences in surgeon preference for the treatment of cervical facet injuries: A case study survey with the AO spine cervical classification validation group. Eur. Spine J. 2021, 30, 517-523. [CrossRef] [PubMed]

113. Mavrovounis, G.; Meling, T.R.; Lafuente, J.; Fountas, K.N.; Demetriades, A.K. Postural ergonomics and work-related musculoskeletal disorders in neurosurgery: Lessons from an international survey. Acta Neurochir. 2021, 163, 1541-1552. [CrossRef] [PubMed]

114. Schroeder, G.D.; Canseco, J.A.; Patel, P.D.; Divi, S.N.; Karamian, B.A.; Kandziora, F.; Vialle, E.N.; Oner, F.C.; Schnake, K.J.; Dvorak, M.F.; et al. Establishing the injury severity of subaxial cervical spine trauma: Validating the hierarchical nature of the ao spine subaxial cervical spine injury classification system. Spine 2021, 46, 649-657. [CrossRef] [PubMed]

115. Montemurro, N.; Santoro, G.; Marani, W.; Petrella, G. Posttraumatic synchronous double acute epidural hematomas: Two craniotomies, single skin incision. Surg. Neurol. Int. 2020, 11, 435. [CrossRef] [PubMed]

116. Ridge, S.E.; Shetty, K.R.; Lee, D.J. Heads-up surgery: Endoscopes and exoscopes for otology and neurotology in the era of the COVID-19 pandemic. Otolaryngol. Clin. N. Am. 2021, 54, 11-23. [CrossRef]

117. Montemurro, N. Intracranial hemorrhage and COVID-19, but please do not forget "old diseases" and elective surgery. Brain Behav. Immun. 2021, 92, 207-208. [CrossRef]

118. Gordon, S.A.; Deep, N.L.; Jethanamest, D. Exoscope and personal protective equipment use for otologic surgery in the era of COVID-19. Otolaryngol. Head Neck Surg. 2020, 163, 179-181. [CrossRef]

119. Haldeman, S.; Nordin, M.; Tavares, P.; Mullerpatan, R.; Kopansky-Giles, D.; Setlhare, V.; Chou, R.; Hurwitz, E.; Treanor, C.; Hartvigsen, J.; et al. Distance management of spinal disorders during the COVID-19 pandemic and beyond: Evidence-based patient and clinician guides from the global spine care initiative. JMIR Public Health Surveill. 2021, 7, e25484. [CrossRef] 